\title{
An active strain electromechanical model for cardiac tissue
}

\author{
F. Nobile ${ }^{1}$, A. Quarteroni ${ }^{1,2, *, \dagger}$ and R. Ruiz-Baier ${ }^{2}$ \\ ${ }^{1}$ MOX_Modellistica e Calcolo Scientifico, Dipartimento di Matematica "F. Brioschi”, Politecnico di Milano, \\ via Bonardi 9, 20133 Milan, Italy \\ ${ }^{2}$ Modeling and Scientific Computing, MATHICSE-SB, École Polytechnique Fédérale de Lausanne, CH-1015,
} Lausanne, Switzerland

\begin{abstract}
SUMMARY
We propose a finite element approximation of a system of partial differential equations describing the coupling between the propagation of electrical potential and large deformations of the cardiac tissue. The underlying mathematical model is based on the active strain assumption, in which it is assumed that there is a multiplicative decomposition of the deformation tensor into a passive and active part holds, the latter carrying the information of the electrical potential propagation and anisotropy of the cardiac tissue into the equations of either incompressible or compressible nonlinear elasticity, governing the mechanical response of the biological material. In addition, by changing from a Eulerian to a Lagrangian configuration, the bidomain or monodomain equations modeling the evolution of the electrical propagation exhibit a nonlinear diffusion term. Piecewise quadratic finite elements are employed to approximate the displacements field, whereas for pressure, electrical potentials and ionic variables are approximated by piecewise linear elements. Various numerical tests performed with a parallel finite element code illustrate that the proposed model can capture some important features of the electromechanical coupling and show that our numerical scheme is efficient and accurate. Copyright () 2011 John Wiley \& Sons, Ltd.
\end{abstract}

Received 9 May 2011; Revised 6 July 2011; Accepted 25 July 2011

KEY WORDS: cardiac electromechanical coupling; bidomain equations; reaction-diffusion problem; active strain; nonlinear elasticity; finite elements

\section{INTRODUCTION}

The mathematical modeling of the complex physical phenomena occurring in the heart is an area of increasing interest, as it facilitates the better understanding of relevant mechanisms driving the behavior of the system in both physiological and pathological contexts. In this paper, we are interested in the study of the interaction between the propagation of the electrical potential through the cardiac tissue and the related mechanical response. Several difficulties and major challenges arise in this context, such as geometrical irregularities, physical nonlinearities, uncertainty of material parameters, and anisotropy of the tissue, to name a few. This subject has gained a considerable attention in recent years, as shown by the large number of contributions in applied mathematics and bioengineering (see, e.g., [1-4] and the references therein). The diversity of these studies suggests that both the modeling and numerical treatment of this class of problems is far from being a resolved subject. A considerable amount of literature is available for the much more established understanding of a particular facet of the problem, namely the mechanisms that drive the electrophysiological activity in the heart. From a scientific computing perspective, a wide class of numerical methods with different degrees of complexity have been proposed and analyzed for efficiently solving the

\footnotetext{
*Correspondence to: A. Quarteroni, Modeling and Scientific Computing, MATHICSE-SB, École Polytechnique Fédérale de Lausanne, CH-1015 Lausanne, Switzerland.

†E-mail: alfio.quarteroni@epfl.ch 
so-called bidomain and monodomain equations modeling the propagation of electrical potentials in the myocardium [5-8].

In this paper, we introduce some advances on a model for cardiac electromechanics, and we propose a suitable numerical method for its approximation. Our model for the excitation-contraction mechanism is inspired by the description in $[9,10]$. The deformation of the tissue is modeled assuming a quasi-steady elasticity framework, in which we suppose that a multiplicative decomposition between the active and passive mechanical response is introduced at the deformation level. This will imply in particular that the fiber contraction driving the depolarization of the tissue rewrites in the mechanical balance of forces as a prescribed active deformation, rather than as an additive contribution to the stresses [1]. Our proposed approach allows a direct incorporation of the micro-level information on the fiber contraction in the kinematics, without the intermediate transcription of their role in terms of the stress. Moreover, in this context, we consider that the active part of the mechanical response also carries the information about the anisotropy of the fibrous tissue architecture, implying an isotropic description for the passive mechanics. Despite some necessary simplifications in the underlying physics, the proposed model is able to address the main features of the complete mechanical/electro-dynamical system, providing more insight on the role of the active strain in the cardiac electromechanical phenomenon. Our framework can of course accommodate the study of more general material properties, such as full orthotropy for the passive mechanical response, and a wider range of model parameters.

A further aims of this paper were to devise an adequate numerical scheme for obtaining stable, efficient, and accurate approximations of the underlying coupled problem and to provide some numerical examples to illustrate the behavior of the phenomenon, which will allow us to discuss the impact of several modeling choices. The resulting nonlinear balance equations are treated using a Newton algorithm, and the corresponding spatial discretization is performed by applying a finite element framework.

The remainder of this paper is organized as follows. In Section 2, the bidomain model for the electrical activity is outlined, followed by a description of an appropriate mechanical framework on the basis of finite elasticity. Next, we give a precise meaning to the coupling between mechanics and electrical activity in the tissue. In Section 3, we construct the corresponding finite element formulation to solve the derived coupled problem, and Section 4 contains several numerical examples that illustrate the good behavior of the models and method proposed. Finally, some conclusions and possible extensions are drawn in Section 5.

\section{FORMULATION OF THE ELECTROMECHANICAL PROBLEM}

A contraction of the cardiac muscle generally takes place in response to an electrical impulse and because of internal activation mechanisms. On the other hand, it is known that myocardial stretch can cause changes in the electrophysiological properties of the heart (meccano-electrical feedback). As a matter of fact, several experimental studies both in vitro and in vivo have proved that the myocardial stretch is responsible for the change in the configuration of action potential, which leads to afterdepolarization-like activity and arrhythmias (see, e.g., [11]).

Roughly speaking, the cardiac electromechanical response behaves as follows. An electrical impulse starts in the sinoatrial node. There, a depolarization begins and a wave propagates across the atria, followed by a delay of the potential at the atrioventricular node. Then, a rapid depolarization of both ventricles occurs, which at the cellular level causes an increase of calcium concentration, and this mechanism produces a contraction by a temporary binding between actin and myosin. In trying to study this complex mechanism, we will focus on the macroscopic aspects of the coupling.

In the following, we divide the description into three main parts: the equations governing the electrical activity, the equations for the mechanical behavior of the tissue, and finally the coupling strategy. In order to consider each sub-problem in a natural approach, we will formulate both the electrical propagation and the nonlinear mechanics in a pure Lagrangian framework. To this end, by $\Omega_{o} \subset \mathbb{R}^{3}$, we will denote the bounded spatial domain in the undeformed equilibrium state. 


\subsection{The governing equations for the electrophysiology}

In this section, we recall the main equations for electrophysiology considered in a fixed mechanical configuration. Their extension to the case of a deforming domain is postponed to Section 2.3. The quantities of interest in the bidomain model for electrical signaling in the heart are the intracellular and extracellular electric potentials, $u_{\mathrm{i}}=u_{\mathrm{i}}(x, t)$ and $u_{\mathrm{e}}=u_{\mathrm{e}}(x, t)$, defined at $(x, t) \in \Omega_{T}:=\Omega \times(0, T)$. Their difference $v=v(x, t):=u_{\mathrm{i}}-u_{\mathrm{e}}$ is the transmembrane potential. The conductivity of the tissue is represented by tensors $\mathbf{D}_{\mathrm{k}}(x)$ given by

$$
\mathbf{D}_{\mathrm{k}}(x)=\sigma_{\mathrm{k}}^{1} \boldsymbol{a}_{1} \otimes \boldsymbol{a}_{1}+\sigma_{\mathrm{k}}^{\mathrm{t}} \boldsymbol{a}_{\mathrm{t}} \otimes \boldsymbol{a}_{\mathrm{t}}+\sigma_{\mathrm{k}}^{\mathrm{n}} \boldsymbol{a}_{\mathrm{n}} \otimes \boldsymbol{a}_{\mathrm{n}} \quad \mathrm{k} \in\{\mathrm{e}, \mathrm{i}\},
$$

where $\sigma_{\mathrm{k}}^{\mathrm{j}} \in C^{1}\left(\mathbb{R}^{3}\right)$ are intracellular and extracellular conductivities along the directions $\boldsymbol{a}_{\mathrm{j}}(x)$, $\mathrm{j} \in\{1, \mathrm{t}, \mathrm{n}\}$, representing a triplet of orthonormal vectors with $\boldsymbol{a}_{1}(x)$ being parallel to the local fibers' direction. Such description is crucial in the model, as the cardiac tissue is actually made of fibers that drive the propagation of the electrical potential.

The bidomain model, introduced by Tung [12], is given by the following coupled reactiondiffusion system

$$
\begin{gathered}
\chi c_{\mathrm{m}} \partial_{t} v-\nabla \cdot\left(\mathbf{D}_{\mathrm{i}}(x) \nabla u_{\mathrm{i}}\right)+\chi I_{\mathrm{ion}}(v, \boldsymbol{w})=I_{\text {app }}^{\mathrm{i}}, \\
\chi c_{\mathrm{m}} \partial_{t} v+\nabla \cdot\left(\mathbf{D}_{\mathrm{e}}(x) \nabla u_{\mathrm{e}}\right)+\chi I_{\text {ion }}(v, \boldsymbol{w})=I_{\text {app }}^{\mathrm{e}}, \\
\partial_{t} \boldsymbol{w}-H(v, \boldsymbol{w})=0, \quad(x, t) \in \Omega_{T},
\end{gathered}
$$

provided with homogeneous Neumann boundary conditions. Here, $c_{\mathrm{m}}>0$ is the surface capacitance of the membrane, $\chi$ is the ratio of membrane area per tissue volume, and $\boldsymbol{w}(x, t)$ is a ionic variable, which essentially controls the local repolarization behavior of the action potential and it is scalar or vectorial, depending on the choice of membrane model. The symbol $\partial_{t}$ stands for the partial derivative with respect to the time variable $t$. The knowledge of suitable initial conditions for $v, u_{\mathrm{e}}, \boldsymbol{w}$ is also required. The stimulation current possibly applied to the extracellular space is represented by the functions $I_{\text {app }}^{\mathrm{k}}=I_{\text {app }}^{\mathrm{k}}(x, t)$. In the case that $\mathbf{D}_{\mathrm{i}}=\varrho \mathbf{D}_{\mathrm{e}}$ for some $\varrho \in \mathbb{R}$, the bidomain system reduces to the so-called monodomain model (see, e.g., [6]):

$$
\begin{gathered}
\chi c_{\mathrm{m}} \partial_{t} v-\nabla \cdot\left(\frac{\mathbf{D}_{\mathrm{i}}(x)}{1+\varrho} \nabla v\right)+\chi I_{\mathrm{ion}}(v, \boldsymbol{w})=\frac{\varrho}{1+\varrho} I_{\mathrm{app}}, \\
\partial_{t} \boldsymbol{w}-H(v, \boldsymbol{w})=0, \quad(x, t) \in \Omega_{T} .
\end{gathered}
$$

This somewhat simpler model requires less computational effort than (2.1), and even though the assumption of equal anisotropy ratios is physiologically inaccurate, (2.2) is still adequate for a qualitative investigation of certain repolarization sequences and the distribution of patterns of durations of the action potential [6].

The choice of the functions $H(v, w)$ and $I_{\text {ion }}(v, w)$ is determined by the membrane model to be employed. Depending on the level of complexity of the problem under investigation, we will restrict ourselves to two of these. First, the adimensional Rogers-McCulloch model [13], which is based mainly on phenomenological evidence, and corresponds to

$$
\begin{aligned}
H(v, w) & =b v-w, \\
I_{\text {ion }}(v, w) & =c_{2} v w-c_{1} v(1-v)(v-a),
\end{aligned}
$$

where $w$ is a scalar gating variable, and $a, b, c_{1}$, and $c_{2}$ are adimensional parameters. This model is able to capture the characteristic shape of the action potential curve only on some specific repolarization stages (Figure 1, top). The phase diagram in Figure 1 (bottom) shows computed trajectories for different initial values of $v_{0}$ and $\boldsymbol{w}_{0}$, which converge to the stable equilibrium state $(0,0)$. In order to provide results on a physiological scale for electrical potentials and time, we conveniently replaced model (2.3) by the dimensionalized equations 

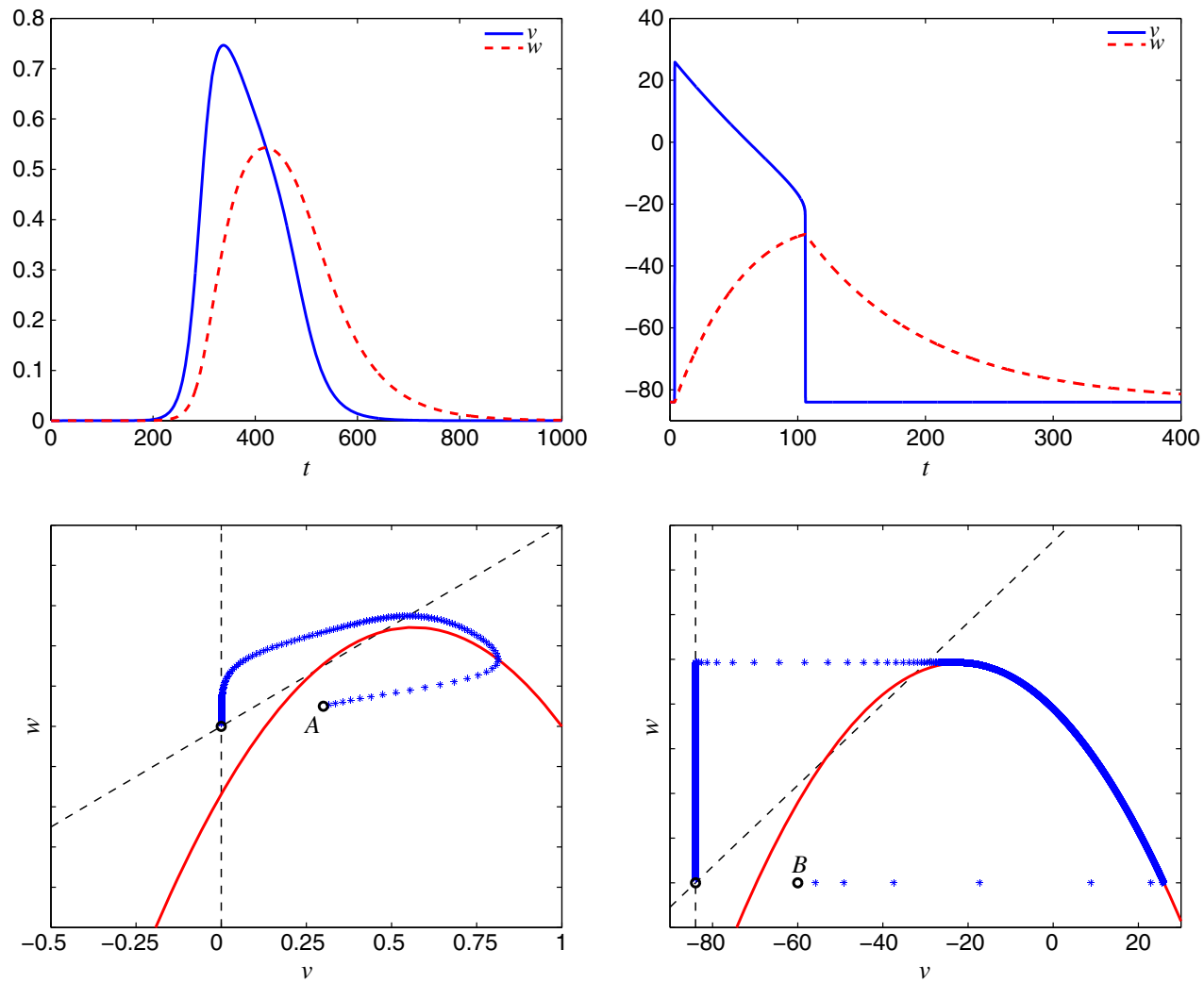

Figure 1. Action potential curve (top) and phase portrait (bottom) for the Rogers-McCulloch model in the adimensional (left) and dimensionalized (right) setting, starting from the initial states $A=(0.3,0.1)$, $B=(-60,0)$ and reaching the intersections of the nullclines.

$$
\begin{aligned}
H(v, w) & =\frac{b}{T A}\left(v-v_{\min }-A w\right), \\
I_{\text {ion }}(v, w) & =\frac{c_{1}}{T A^{2}}\left(v-v_{\min }\right)\left(v-v_{\min }-a A\right)\left(v-v_{\min }-A\right)+\frac{c_{2}}{T}\left(v-v_{\min }\right) w .
\end{aligned}
$$

The right column of Figure 1 displays the corresponding action potential curves and phase diagram. Secondly, we use the phase I Luo-Rudy (LRI) model [14] constructed on the basis of a description of ionic currents. In that model, $\boldsymbol{w}$ is a vector of dimensionless ion-channel gating variables, and the total ionic current density $I_{\text {ion }}(v, w)$ is the sum of a fast inward sodium current $I_{\mathrm{Na}}$, a slow inward current $I_{\mathrm{si}}$, a time-dependent potassium slow outward current $I_{\mathrm{K}}$, an outward potassium current $I_{\mathrm{K}_{1}}$, a plateau potassium current $I_{\mathrm{K}_{\mathrm{p}}}$, and a total background current $I_{\mathrm{b}}$ (see details in, e.g., $[14,15])$.

\subsection{Model for finite elasticity}

The myocardium is composed of connective tissue and cells surrounded by space filled with fluid, all of these materials being mainly formed by water. From the mechanical viewpoint, the heart tissue in its resting state can be regarded as an inhomogeneous, anisotropic, and incompressible (or nearly incompressible) elastic material [16]. The tissue is subject to external load and active deformation inducing a strain field. An adequate scenario for the modeling of cardiac mechanics is then provided by the nonlinear elasticity framework.

From now on, $\boldsymbol{x} \in \Omega$ will denote the current position of a particle that occupied the position $\mathbf{X} \in \Omega_{o}$ in the initial undeformed configuration, and $\boldsymbol{u}=\boldsymbol{x}-\mathbf{X}$ will stand for the displacement field. Then, 


$$
\mathbf{F}=\nabla \boldsymbol{x}=\mathbf{I}+\nabla \boldsymbol{u}, \quad F_{i j}=\frac{\partial x_{i}}{\partial X_{j}}=\delta_{i j}+\frac{\partial \boldsymbol{u}_{i}}{\partial X_{j}},
$$

where $\delta_{i j}$ denotes the Kronecker delta and is the deformation gradient tensor, measuring strain between the deformed and undeformed states. The symbol $\nabla$ denotes the gradient of a quantity with respect to the material coordinates $\mathbf{X}$. We assume that $\mathbf{F}$ can be decomposed (factorized) into an elastic (passive) factor, taking place at a macroscale, and an active deformation gradient tensor, acting at a microscale in the following form [10]

$$
\mathbf{F}=\mathbf{F}_{e} \mathbf{F}_{a} .
$$

Such decomposition assumes that an intermediate elastic configuration $\Omega_{e}$ exists between the reference state $\Omega_{o}$ and the current loaded configuration $\Omega$.

Notice that $\mathbf{F}$ is given by the gradient of a vector map whereas $\mathbf{F}_{e}, \mathbf{F}_{a}$ are not, in general. In the sequel, we will refer to this setting as the active strain formulation. Similar decompositions have been proposed in the context of finite elastoplasticity [17] and growth modeling [18].

By $J, J_{a}$, we denote the determinants of $\mathbf{F}, \mathbf{F}_{a}$, respectively. The Jacobian $J$ describes the volume map of infinitesimal reference elements onto the corresponding current elements. In other electromechanical models available (see, e.g., $[2-4,19]$ ), an appropriate term is added to the passive stress tensor, generating an additive decomposition between passive and active stress. We will refer to the latter decomposition as active stress formulation. It is demonstrated in [10] that the active stress decomposition is equivalent to (2.5) only in the special case of small deformations.

The time-space scales in the cardiac electromechanical phenomenon suggest the use of steadystate equations of motion to describe the conservation of linear and angular momentum [3]. These are reduced to the force balance

$$
-\nabla \cdot \mathbf{P}=\boldsymbol{f},
$$

where $\mathbf{P}$ is the Piola-Kirchhoff stress, which represents force per unit undeformed surface, and $\boldsymbol{f}$ is a vector of body forces. Note that the balance is defined in the undeformed state $\Omega_{o}$.

The definition of $\mathbf{P}$ in terms of the components of the deformation stress and strain measures is given by the constitutive relations. In the context under study, the medium is typically assumed to be a hyperelastic material. Therefore, it can be postulated that there exists an elastic strain energy density function $\mathcal{W}=\mathcal{W}(\mathbf{F})$ defined in the reference configuration and depending only on the value of the deformation gradient, which characterizes the material. Notice that the energy in the intermediate elastic configuration is

$$
\mathcal{W}_{e}=\mathcal{W}\left(\mathbf{F}_{e}\right)=\mathcal{W}\left(\mathbf{F F}_{a}^{-1}\right),
$$

and therefore a pull back to the reference state gives a new energy

$$
\widehat{\mathcal{W}}=J_{a} \mathcal{W}\left(\mathbf{F F}_{a}^{-1}\right) .
$$

We point out that if the chosen strain energy function has desirable stability properties (such as polyconvexity and coercivity, as discussed in, e.g., [16]), then the application of an active strain decomposition like (2.5) essentially translates into a natural shift of the relaxation state from I to $\mathbf{F}_{a}^{-1}$, therefore preserving the qualitative structure of $\mathcal{W}$. In this sense, the active strain formulation can be straightforwardly extended to the study of more adequate material laws, such as structurally based models that account for the passive properties of the cardiac tissue.

For the derivation of the full model, we start by considering a Neo-Hookean material, for which the internal stored energy function in the intermediate configuration reads

$$
\mathcal{W}\left(\mathbf{F}_{e}\right)=\frac{\mu_{1}}{2} \operatorname{tr}\left(\mathbf{F}_{e}^{T} \mathbf{F}_{e}-\mathbf{I}\right),
$$

where $\mu_{1}$ is a shear modulus. To assure incompressibility of the material (where only isochoric behavior is allowed), we assumed the strain energy to take the form 


$$
\widehat{\mathcal{W}}=J_{a} \mathcal{W}\left(\mathbf{F}_{e}\right)-p(J-1)
$$

where $p$ is the Lagrange multiplier arising from the imposition of the incompressibility constraint $J=1$ (conservation of mass) and which is usually interpreted as the hydrostatic pressure field.

The Piola-Kirchhoff stress tensor is given by the Frechet derivative of the internal stored energy function $\mathcal{W}$, which in the fully relaxed configuration reads

$$
\mathbf{P}=J_{a} \frac{\partial \mathcal{W}}{\partial \mathbf{F}_{e}} \mathbf{F}_{a}^{-T}-p \mathbf{F}_{e}^{-T} \mathbf{F}_{a}^{-T}
$$

An alternative step considered here is the assumption of nearly incompressible materials, which in turn allows to avoid solving a saddle-point-like problem. In such case, a suitable strain energy function for Neo-Hookean materials is [20]

$$
\mathcal{W}\left(\mathbf{F}_{e}\right)=\frac{\mu_{1}}{2} J_{a} \operatorname{tr}\left(\mathbf{F}_{e}^{T} \mathbf{F}_{e}-\mathbf{I}\right)+\frac{\mu_{2}}{2}(J-1)^{2}-\mu_{1} J_{a} \ln (J),
$$

if $J>0$, and $\mathcal{W}\left(\mathbf{F}_{e}\right)=\infty$ otherwise. Here, $\mu_{2}$ is a bulk modulus. The discussion on whether the myocardium should be modeled as incompressible or nearly incompressible is apparently not resolved; we therefore leave the door open for considering both approaches.

Notice that in the material law used herein, so far we have not addressed a major feature in the modeling of cardiac dynamics: the anisotropy of the tissue. Obviously, the strain energy could also be assumed to depend explicitly on the fibers distribution through the inclusion of further invariants of the left Cauchy-Green tensor or through components of the Green-Lagrange strain tensor, as in, for example, [3,21,22].

In this work, we follow a simplified approach and, as an intermediate step, propose to account for the anisotropic behavior of the fibers only by assigning direction-specific active deformation fields in the active part of the decomposition. That is, we assume for the moment that the passive elastic response is isotropic. More specifically, for a myofiber distribution along the direction of the unit vectors $\boldsymbol{a}_{1}, \boldsymbol{a}_{\mathrm{t}}$, we consider that the active strain assumes the form

$$
\mathbf{F}_{a}=\mathbf{I}+\gamma_{1} \boldsymbol{a}_{1} \otimes \boldsymbol{a}_{1}+\gamma_{\mathrm{t}} \boldsymbol{a}_{\mathrm{t}} \otimes \boldsymbol{a}_{\mathrm{t}},
$$

where $\boldsymbol{a}_{1}, \boldsymbol{a}_{\mathrm{t}}$ are the fiber sheet longitudinal and transversal directions respectively, and $\gamma_{i}$ are scalar fields accounting for the activation, depending on macroscopic stimuli related to the electrical part of the model, which will be made precise later. Given the special constitutive form of $\mathbf{F}_{a}$, under transverse anisotropy, we can readily write

$$
J_{a}=\left(1+\gamma_{1}\right)\left(1+\gamma_{\mathrm{t}}\right)
$$

Putting together the previous description, we obtain that the Euler-Lagrange problem (in its weak formulation) reads as follows: find $\boldsymbol{u}, p$ in suitable admissible displacement and pressure spaces such that

$$
\begin{array}{r}
\int_{\Omega_{o}}\left(\mu_{1} J_{a}(\mathbf{I}+\nabla \boldsymbol{u}) \mathbf{F}_{a}^{-1} \mathbf{F}_{a}^{-T}: \nabla \boldsymbol{\varphi}-p J(\mathbf{I}+\nabla \boldsymbol{u})^{-T}: \nabla \boldsymbol{\varphi}\right)=0 \\
\int_{\Omega_{o}}(J-1) q=0,
\end{array}
$$

for all test functions $\boldsymbol{\varphi}, q$ in the same spaces as $\boldsymbol{u}$ and $p$, respectively.

\subsection{The coupled model}

With the purpose of studying the basic mechanisms of the meccano-electrical feedback, and the related numerical challenges, we herein consider a coupled model in which we retain only the most essential elements. We first assume that the active deformation functions $\gamma_{\mathrm{j}}, \mathrm{j} \in\{1, \mathrm{t}\}$ 
(Figure 2, where $\gamma_{\mathrm{j}}$ has been rescaled for visualization purposes), depend directly on the transmembrane potential through the following saturation-like relation:

$$
\gamma_{1}(v)=-\beta \frac{v-v_{\min }}{v_{\max }-v_{\min }+v}, \quad \gamma_{\mathrm{t}}(v)=\frac{-\gamma_{1}(v)}{1+\gamma_{1}(v)},
$$

where $v_{\min }, v_{\max }$ are problem-dependent parameters accounting for the proper scaling of the solution, and $\beta=0.3$ is included to model the change of length experimented by the cardiac fibers in a normal heartbeat. The definition of $\gamma_{1}$ implies that the contraction of a slab of tissue in the fibers' direction is of opposite sign to that in the direction $\boldsymbol{a}_{\mathrm{t}}$. The particular form of $\gamma_{\mathrm{t}}$ obeys to the assumption that the active deformation is also volume preserving: $J_{a}=1$. Notice that $\gamma_{1}<0$ implies a contraction of the myocardium. This approach then assumes that the information of the electrical part of the model enters in the mechanical description through (2.6) and (2.8) only. To account for the coupling at the microscale, we also relate the activation function in the fibers' direction $\gamma_{1}$ to the intracellular calcium concentration $[\mathrm{Ca}]_{+}$in the following way:

$$
\gamma_{1}\left(v,[\mathrm{Ca}]_{+}\right)=-\beta \frac{v-v_{\min }}{v_{\max }-v_{\min }+v}+\varepsilon_{1} \beta \frac{l_{o}^{l}}{1+\eta\left([\mathrm{Ca}]_{+}\right)\left(l_{o}^{l}-1\right)},
$$

where $l_{o}^{l}=\left(\eta\left(c_{o}^{*}\right)-\varepsilon_{1}\right)^{-1}\left(\eta\left(c_{o}^{*}\right)-1\right), \eta\left([\mathrm{Ca}]_{+}\right)=\frac{1}{2}+\frac{1}{\pi} \arctan \left(\beta^{2} \log \left([\mathrm{Ca}]_{+} / c_{R}\right)\right)$, and $c_{o}^{*}, c_{R}, \varepsilon_{1}$ are given parameters (see [1], where the authors propose the last term in (2.9). We add also the first term to include the direct influence of the transmembrane potential). We employ (2.9) only when a ionic model (such as Luo-Rudy) is considered. For simplified membrane models (RogersMcCulloch), the terms $\gamma_{i}$ are taken as in (2.8). We also consider a more biophysically detailed model for the active contraction, in which the function $\gamma_{1}$ is defined as the development of tension from cellular cross-bridge cycling, and its evolution is modeled using a system of ODEs.

The ODE system modeling the kinetics of the membrane and of the activation $\gamma_{1}$ is given by [23]

$$
\partial_{t} v=I_{\text {ion }}(v, \boldsymbol{w}), \quad \partial_{t} \boldsymbol{w}=H(v, \boldsymbol{w}), \quad \partial_{t} \gamma_{1}=G\left(\boldsymbol{w}, \gamma_{1}\right) .
$$

For the Rogers-McCulloch model, $G$ in (2.10) is

$$
G\left(w, \gamma_{1}\right)=d_{1}^{\mathrm{RM}}\left(-\beta w-d_{2}^{\mathrm{RM}} \gamma_{1}\right),
$$

whereas for the Luo-Rudy I model, it is

$$
G\left(\boldsymbol{w}, \gamma_{1}\right)=d_{1}^{\mathrm{LR}}\left(-\beta[C a]_{i}^{+}-d_{2}^{\mathrm{LR}} \gamma_{1}\right),
$$

where the parameters are $d_{1}^{\mathrm{RM}}=0.025, d_{2}^{\mathrm{RM}}=0.42, d_{1}^{\mathrm{LR}}=2.2$, and $d_{2}^{\mathrm{LR}}=0.005$. Figure 3 depicts the time evolution for (2.10) computed on a single cell in which the amplitudes of both calcium concentration and activation have been rescaled for visualization purposes. We see a qualitative agreement with the numerical and experimental results in, for example, [24].
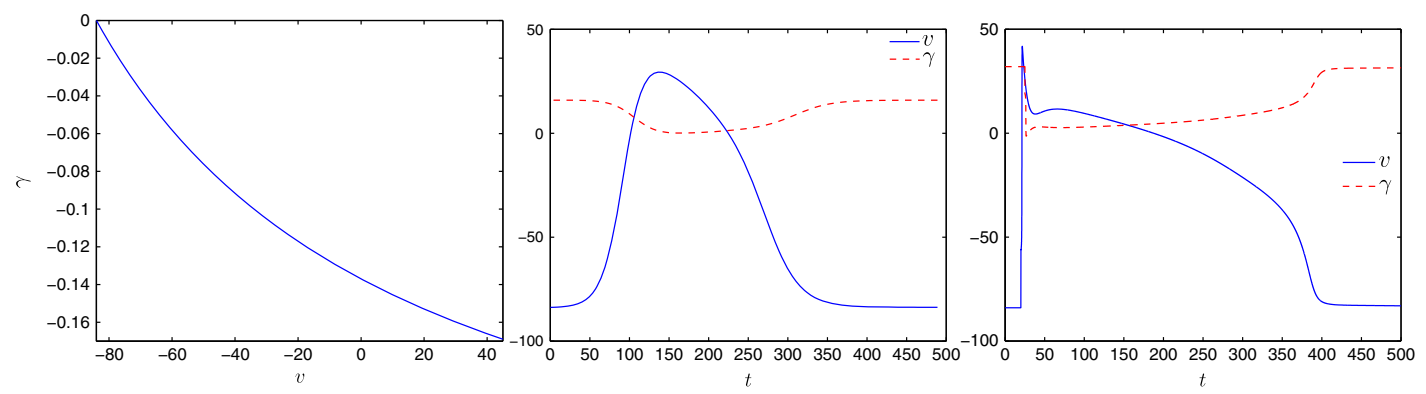

Figure 2. Active deformation function $\gamma=\gamma_{1}$ (a measure of the active strain in the direction of the fibers) depending on the transmembrane potential (left), action potential curve and active deformation function during a period of $500 \mathrm{~ms}$ for the Rogers-McCulloch and Luo-Rudy I models (middle and right, respectively). 

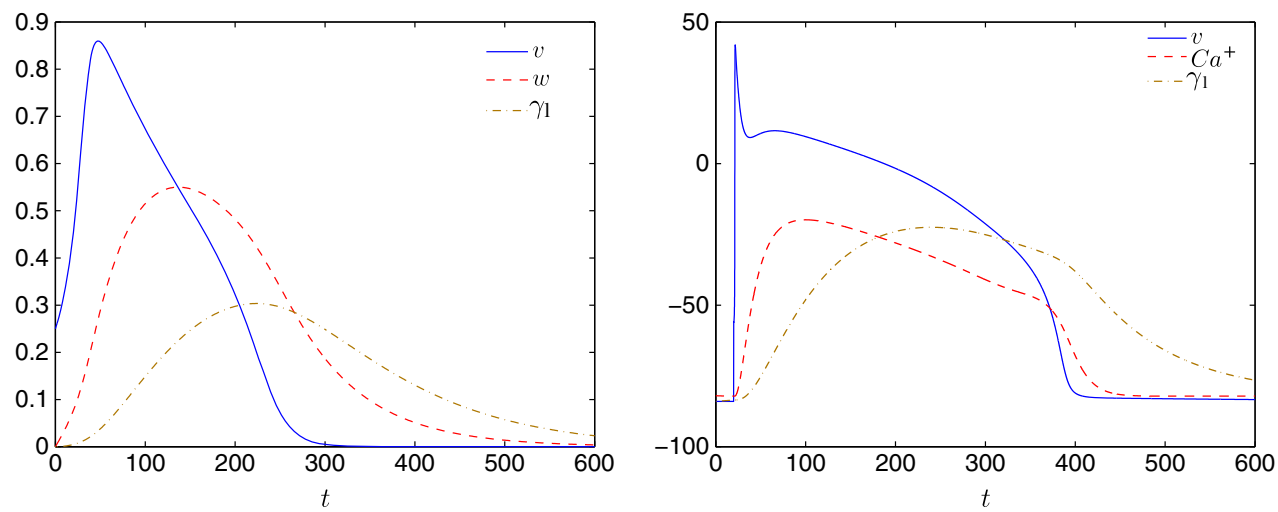

Figure 3. Evolution of the active deformation function $\gamma_{1}$, action potential, and calcium concentration, when computed from system (2.10). Rogers-McCulloch and Luo-Rudy I models (left and right, respectively).

The second main ingredient of the electromechanical coupling is the proper representation of the dependence of the electrical properties of the tissue, on the active strain. In our model, the influence of the mechanical response into the electrophysiology is provided by a transformation of coordinates from Eulerian to Lagrangian and the use of the Piola identity $\nabla \cdot\left(J \mathbf{F}^{-T}\right)=0$ (as in [9]). Other related approaches (to be included in a forthcoming analysis) are based on a dependence of ionic currents, on certain microscopic mechanical processes [4,22]. Collecting the items of the analysis above, and considering for instance, the case of incompressible materials, we end up with the following bidomain electromechanical coupled model:

$$
\begin{aligned}
& -\nabla \cdot\left(\mu_{1} J_{a}(\mathbf{I}+\nabla \boldsymbol{u}) \mathbf{F}_{a}^{-1} \mathbf{F}_{a}^{-T}-p J(\mathbf{I}+\nabla \boldsymbol{u})^{-T}\right)=0 \text { in } \Omega_{o}, \\
& J=1 \text { in } \Omega_{o}, \\
& \chi c_{\mathrm{m}} \partial_{t}(J v)-\nabla \cdot\left(J(\mathbf{I}+\nabla \boldsymbol{u})^{-1} \mathbf{D}_{\mathrm{e}}(\mathbf{I}+\nabla \boldsymbol{u})^{-T} \nabla u_{\mathrm{e}}\right)+\chi J I_{\text {ion }}=J I_{\text {app }}^{\mathrm{i}} \quad \text { in } \Omega_{T}, \\
& \chi c_{\mathrm{m}} \partial_{t}(J v)+\nabla \cdot\left(J(\mathbf{I}+\nabla \boldsymbol{u})^{-1} \mathbf{D}_{\mathrm{i}}(\mathbf{I}+\nabla \boldsymbol{u})^{-T} \nabla u_{\mathrm{i}}\right)+\chi J I_{\text {ion }}=J I_{\text {app }}^{\mathrm{e}} \quad \text { in } \Omega_{T}, \\
& \partial_{t}(J \boldsymbol{w})-J H(v, \boldsymbol{w})=0 \quad \text { in } \Omega_{T}, \\
& \partial_{t}\left(J \gamma_{1}\right)-J G\left(\boldsymbol{w}, \gamma_{1}\right)=0 \quad \text { in } \Omega_{T} .
\end{aligned}
$$

The system of equations has to be completed with no-flux boundary conditions for the electric variables, suitable initial data for $u_{\mathrm{e}}, v, \boldsymbol{w}$, and we specify a displacement field on a portion $\Gamma_{D}$ of $\partial \Omega_{o}$, which in particular has been taken as homogeneous Dirichlet boundary data

$$
\boldsymbol{u}=\mathbf{0} \quad \text { on } \Gamma_{D} \subset \partial \Omega_{o},
$$

and we impose homogeneous Neumann conditions

$$
\mathbf{P n}=\mathbf{0},
$$

on $\partial \Omega_{o} \backslash \Gamma_{D}$.

Analogously, we have the monodomain electromechanical system (written in terms of $v, p, \mathbf{X}$ ):

$$
\begin{array}{rlrl}
-\nabla \cdot\left(\mu_{1} J_{a}(\mathbf{I}+\nabla \boldsymbol{u}) \mathbf{F}_{a}^{-1} \mathbf{F}_{a}^{-T}-p J(\mathbf{I}+\nabla \boldsymbol{u})^{-T}\right) & =0 & & \text { in } \Omega_{o}, \\
J & =1 & \text { in } \Omega_{o}, \\
\chi c_{\mathrm{m}} \partial_{t}(J v)-\nabla \cdot\left(J(\mathbf{I}+\nabla \boldsymbol{u})^{-1} \mathbf{D}(\mathbf{I}+\nabla \boldsymbol{u})^{-T} \nabla v\right)+J I_{\text {ion }}(v, w) & =\varrho /(1+\varrho) J I_{\text {app }} & \text { in } \Omega_{T}, \\
\partial_{t}(J w)-J H(v, w) & =0 & & \text { in } \Omega_{T}, \\
\partial_{t}\left(J \gamma_{1}\right)-J G\left(w, \gamma_{1}\right) & =0 & & \text { in } \Omega_{T} .
\end{array}
$$

Note that the third equation is a balance equation for the potential, accounting for the dynamics between flow and reactions of ionic species, whereas the last equations are pointwise attached to the material and do not represent any balance in space. Note also that (2.11) and (2.14) are both written in the total Lagrangian formulation, that is, only in terms of the undeformed configuration. 


\subsection{Weak formulation}

Let us denote $H_{D}^{1}\left(\Omega_{o}\right)=\left\{s \in H^{1}\left(\Omega_{o}\right):\left.s\right|_{\Gamma_{D}}=0\right\}$. Assuming that all unknowns are regular enough (we suppose $v, u_{\mathrm{e}} \in L^{2}\left(0, T ; H^{1}\left(\Omega_{o}\right)\right), w \in L^{2}\left(0, T, L^{2}\left(\Omega_{o}\right)\right), \boldsymbol{u} \in L^{2}\left(0, T ; H_{D}^{1}\left(\Omega_{o}\right)^{3}\right)$, $p \in L_{0}^{2}\left(\Omega_{o}\right)$, in order to get bounded energy integrals), we multiply the equations in (2.11) by a vectorial test field $\varphi$ vanishing on $\Gamma_{D}$ and by scalar test functions $q, \xi_{\mathrm{i}}, \xi_{\mathrm{e}}, \xi, \psi$, respectively. The weak problem associated to the coupled electromechanical model (2.11) reads as follows. Given $v_{0}, w_{0} \in L^{2}\left(\Omega_{o}\right), I_{\text {app }} \in L^{2}\left(\Omega_{T}\right)$, for $t \in(0, T)$, find a displacement vector $\boldsymbol{u}$, pressure $p$, electrical potentials $v, u_{\mathrm{e}}$, ionic variables $\boldsymbol{w}$, and activation $\gamma_{1}$ such that the following identities hold for all test functions $\varphi, q, \xi_{\mathrm{j}}, \xi, \psi$ :

$$
\begin{aligned}
\mu_{1} \int_{\Omega_{o}}(\mathbf{I}+\nabla \boldsymbol{u}) J_{a} \mathbf{F}_{a}^{-1} \mathbf{F}_{a}^{-T}: \nabla \boldsymbol{\varphi}-\int_{\Omega_{o}} p J(\mathbf{I}+\nabla \boldsymbol{u})^{-T}: \nabla \boldsymbol{\varphi} & =0, \\
\int_{\Omega_{o}}(J-1) q & =0, \\
\int_{\Omega_{o}} \chi c_{\mathrm{m}} \partial_{t}(J v) \xi_{\mathrm{i}}+\int_{\Omega_{o}} J(\mathbf{I}+\nabla \boldsymbol{u})^{-1} \mathbf{D}_{\mathrm{i}}(\mathbf{I}+\nabla \boldsymbol{u})^{-T} \nabla u_{\mathrm{i}} \cdot \nabla \xi_{\mathrm{i}}+\chi \int_{\Omega_{o}} J I_{\mathrm{ion}} \xi_{\mathrm{i}} & =\int_{\Omega_{o}} J I_{\mathrm{app}}^{\mathrm{i}} \xi_{\mathrm{i}}, \\
\int_{\Omega_{o}} \chi c_{\mathrm{m}} \partial_{t}(J v) \xi_{\mathrm{e}}-\int_{\Omega_{o}} J(\mathbf{I}+\nabla \boldsymbol{u})^{-1} \mathbf{D}_{\mathrm{e}}(\mathbf{I}+\nabla \boldsymbol{u})^{-T} \nabla u_{\mathrm{e}} \cdot \nabla \xi_{\mathrm{e}}+\chi \int_{\Omega_{o}} J I_{\mathrm{ion}} \xi_{\mathrm{e}} & =\int_{\Omega_{o}} J I_{\mathrm{app}}^{\mathrm{e}} \xi_{\mathrm{e}}, \\
\int_{\Omega_{o}} \partial_{t}(J \boldsymbol{w}) \xi & =\int_{\Omega_{o}} J H \xi, \\
\int_{\Omega_{o}} \partial_{t}\left(J \gamma_{1}\right) \psi & =\int_{\Omega_{o}} J G \psi .
\end{aligned}
$$

Although the mathematical analysis of the bidomain equations for a (restricted) class of membrane models has received several recent contributions (see, e.g., [25, 26]), the solvability analysis of the cardiac mechanical response has been much less studied [27]. As for the electromechanical coupling, it seems that there are no available results in terms of well-posedness and stability of solutions. For the model proposed herein, an analysis of existence (and uniqueness, under additional regularity restrictions on the mechanical variables) of solution, along with the stability of the coupled system, is currently under development [28].

\section{FINITE ELEMENT APPROXIMATION}

In this section, we outline the numerical strategy adopted to discretize (2.11) and to obtain the corresponding approximate solutions.

\subsection{Space-time discretization}

Let $(0, T)$ be partitioned into $\tilde{N}$ subintervals $\left[t^{n}, t^{n+1}\right]$ of constant time step $\Delta t=t^{n+1}-t^{n}$ and denote with a superscript $n$ the quantities computed at time $t^{n}$. Define $I_{\text {ion }}^{n+1}=I_{\text {ion }}\left(v^{n}, \boldsymbol{w}^{n+1}\right)$.

Analogously to previous approaches for the numerical treatment of the multiscale electromechanical coupling (as, e.g., [2,3,21]), here the fully coupled problem will be solved in a segregated (or modular) way and applying a standard backward Euler time integration scheme for ionic variables. However, further efforts are being made to include a monolithic treatment of the coupling, as was carried out in [29]. A summary of the time-stepping algorithm is as follows: assume that all field variables are known at time $t^{n}$. Then,

(i) The activation deformation $\mathbf{F}_{a}^{n}$ is evaluated.

(ii) The displacements $\boldsymbol{u}^{n+1}$ and pressure $p^{n+1}$ are computed from the elasticity model with known active deformation (see details in Section 3.2).

(iii) The ionic variables $\boldsymbol{w}^{n+1}$ are obtained from the previous electrical potential $v^{n}$.

(iv) The new electrical potentials $v^{n+1}, u_{\mathrm{e}}^{n+1}$ are determined in the reference configuration by a pull back that depends on $\boldsymbol{u}^{n+1}$. 
The detailed semidiscrete system related to (2.11) reads as follows: find $\left(\boldsymbol{u}, u_{\mathrm{e}}, v, \boldsymbol{w}, \gamma_{1}\right)^{n+1}$ such that for all $n \in\{1, \ldots, \tilde{N} \Delta t\}$

$$
\begin{gathered}
\int_{\Omega_{o}} \mu_{1}\left(\mathbf{I}+\nabla \boldsymbol{u}^{n+1}\right)\left(\mathbf{F}_{a}^{n}\right)^{-1}\left(\mathbf{F}_{a}^{n}\right)^{-T}: \nabla \boldsymbol{\varphi}-\int_{\Omega_{o}} p^{n+1}\left(\mathbf{I}+\nabla \boldsymbol{u}^{n+1}\right)^{-T}: \nabla \boldsymbol{\varphi}+\int_{\Omega_{o}}\left(J^{n+1}-1\right) q=0, \\
\frac{1}{\Delta t} \int_{\Omega_{o}}\left(\boldsymbol{w}^{n+1}-\boldsymbol{w}^{n}\right) \xi-\int_{\Omega_{o}} H\left(v^{n}, \boldsymbol{w}^{n+1}\right) \xi=0, \\
\frac{1}{\Delta t} \int_{\Omega_{o}}\left(\gamma_{1}^{n+1}-\gamma_{1}^{n}\right) \psi-\int_{\Omega_{o}} G\left(\boldsymbol{w}^{n}, \gamma_{1}^{n+1}\right) \psi=0, \\
\quad+\int_{\Omega_{o}}\left(\chi I_{\mathrm{ion}}^{n+1}-I_{\mathrm{app}}^{\mathrm{i}, n+1}\right) \xi_{\mathrm{i}}=0, \\
\frac{\chi c_{\mathrm{m}}}{\Delta t} \int_{\Omega_{o}}\left(v^{n+1}-v^{n}\right) \xi_{\mathrm{i}}+\int_{\Omega_{o}}\left(\mathbf{I}+\nabla \boldsymbol{u}^{n+1}\right)^{-1} \mathbf{D}_{\mathrm{i}}\left(\mathbf{I}+\nabla \boldsymbol{u}^{n+1}\right)^{-T} \nabla u_{\mathrm{i}}^{n+1} \cdot \nabla \xi_{\mathrm{i}} \\
\quad+\int_{\Omega_{o}}\left(\chi I_{\mathrm{ion}}^{n+1}-I_{\mathrm{app}}^{\mathrm{e}, n+1}\right) \xi_{\mathrm{e}}=0, \\
\frac{\chi c_{\mathrm{m}}}{\Delta t} \int_{\Omega_{o}}\left(v^{n+1}-v^{n}\right) \xi_{\mathrm{e}}-\int_{\Omega_{o}}\left(\mathbf{I}+\nabla \boldsymbol{u}^{n+1}\right)^{-1} \mathbf{D}_{\mathrm{e}}\left(\mathbf{I}+\nabla \boldsymbol{u}^{n+1}\right)^{-T} \nabla u_{\mathrm{e}}^{n+1} \cdot \nabla \xi_{\mathrm{e}}
\end{gathered}
$$

and a similar system is provided, corresponding to the semidiscrete counterpart for (2.14). Notice that we have applied the incompressibility constraint $J=1$ and the constitutive choice $J_{a}=1$ in all terms, except for the last term in the left hand side of (3.1).

As for the spatial discretization, we partition the domain $\Omega_{o}$ using a regular mesh $\mathcal{T}_{h}$ constructed by closed triangles (or tetrahedra for the 3D case) with boundary $\partial K$ and diameter $h_{K}$. The mesh parameter is $h=\max _{K \in \mathcal{T}_{h}}\left\{h_{K}\right\}$, and we consider classical finite element spaces $V_{h}^{r}$ approximating $H^{1}\left(\Omega_{o}\right)$ by piecewise polynomials of maximum order $r$ on $\mathcal{T}_{h}$. More precisely,

$$
V_{h}^{r}=\left\{v \in H^{1}\left(\Omega_{o}\right) \cap C^{0}\left(\overline{\Omega_{o}}\right):\left.v\right|_{K} \in \mathbb{P}_{r}(K) \text { for all } K \in \mathcal{T}_{h}\right\},
$$

for which $\left\{\varphi_{h}^{r}\right\}$ is a basis. It is evident that we are dealing with a saddle-point type problem. Then, for the scheme to formally satisfy the discrete inf-sup or Ladyzhenskaya-Babuska-Brezzi stability condition [30], the displacement field will be approximated using the FE space $V_{h}^{2}$, whereas the pressure (in the incompressible formulation) and electrical potential fields will be approximated using $V_{h}^{1}$, other options being certainly possible (see [31] for a comparison of several discretization methods applied to soft tissue mechanics). The linear systems associated to the bidomain (or monodomain) and ionic subproblems are solved using a preconditioned generalized minimal residual iterative method (with LU preconditioner). On the other hand, the linear systems involved in the Newton step associated to the mechanical problem are solved with the unsymmetric multi-frontal method (UMFPACK).

From a practical point of view, the implementation of the active strain formulation (3.1) differs from a standard finite elasticity problem only by the presence of the term $\mathbf{F}_{a}^{-1} \mathbf{F}_{a}^{-T}$ (in our algorithm evaluated at the previous time step). More generally, also for other commonly used nonlinear elastic models, the active strain contraction can be added without much effort, provided that the balance equations are written in a pull back from the intermediate configuration.

\subsection{Newton iteration}

The non-linear system of equations resulting from the discretization at every time step of the bulk balance equation (2.7)-(2.13) is solved using an incremental iterative Newton-Raphson solution 
procedure. Dropping the superscript denoting time discretization, we denote the solution at the (sub)iteration step $k$ by $\left(\mathbf{F}^{k}, p^{k}\right)$ and the incremental growth of the discrete deformation and pressure by $\delta \mathbf{F}^{k+1}=\mathbf{I}+\delta\left(\nabla \boldsymbol{u}^{k+1}\right), \delta p^{k+1}$. As the convergence behavior of Newton's iterations is known to depend on its proper initialization, as initial guess for the iteration process, we use $\mathbf{F}^{0}=\mathbf{I}$ (the identity matrix), that is, we start from the undeformed geometry. Next, when evolving in time, as initial guess, we take the deformation at the previous time step. The problem in its weak form reads as follows.

Given an approximation of the solution to (2.7)-(2.13) on the sub-iteration step $k$, find $\delta \boldsymbol{u}^{k+1}, \delta p^{k+1}$ in an appropriate space of variations $\left(\left[H_{D}^{1}\left(\Omega_{o}\right)\right]^{d}\right.$ and $L^{2}\left(\Omega_{o}\right)$ for $\delta \boldsymbol{u}^{k+1}$ and $\delta p^{k+1}$, respectively), such that

$$
\begin{aligned}
& \int_{\Omega_{o}} \mu_{1} \nabla\left(\delta \boldsymbol{u}^{k+1}\right) \mathbf{F}_{a}^{-1} \mathbf{F}_{a}^{-T}: \nabla \boldsymbol{\varphi}+p^{k}\left[\left(\mathbf{I}+\nabla \boldsymbol{u}^{k}\right)^{-1} \nabla\left(\delta \boldsymbol{u}^{k+1}\right)\right]^{T}:\left(\mathbf{I}+\nabla \boldsymbol{u}^{k}\right)^{-1} \nabla \boldsymbol{\varphi} \\
& -\delta p^{k+1} \operatorname{Cof}\left(\mathbf{I}+\nabla \boldsymbol{u}^{k}\right): \nabla \boldsymbol{\varphi}+\int_{\Omega_{o}} \mu_{1}\left(\mathbf{I}+\nabla \boldsymbol{u}^{k}\right) \mathbf{F}_{a}^{-1} \mathbf{F}_{a}^{-T}: \nabla \boldsymbol{\varphi} \\
& \quad-\operatorname{Cof}\left(\mathbf{I}+\nabla \boldsymbol{u}^{k}\right): \nabla \boldsymbol{\varphi} p^{k}=0 \\
& \int_{\Omega_{o}} \operatorname{Cof}\left(\mathbf{I}+\nabla \boldsymbol{u}^{k}\right): \nabla\left(\delta \boldsymbol{u}^{k+1}\right) q+\int_{\Omega_{o}}\left(J^{k}-1\right) q=0,
\end{aligned}
$$

for all test functions $\boldsymbol{\varphi}, q$ belonging to the same space of variations. Here, $\operatorname{Cof}(\mathbf{M})$ denotes the matrix of cofactors of the generic tensor $\mathbf{M}$, and $\mathbf{F}_{a}$ does not have superscript as it is taken at the previous time step. Notice that we have used the relation

$$
D \mathbf{F}^{-T}(\delta \boldsymbol{u})=-\mathbf{F}^{-T}(\nabla(\delta \boldsymbol{u}))^{T} \mathbf{F}^{-T}, \text { for all } \delta \boldsymbol{u} .
$$

The stopping criterion for the algorithm has been chosen as follows:

$$
\frac{\left\|\delta \boldsymbol{u}^{k+1}\right\|_{H^{1}\left(\Omega_{o}\right)}}{\left\|\boldsymbol{u}^{k+1}\right\|_{H^{1}\left(\Omega_{o}\right)}}+\frac{\left\|\delta p^{k+1}\right\|_{L^{2}\left(\Omega_{o}\right)}}{\left\|p^{k+1}\right\|_{L^{2}\left(\Omega_{o}\right)}}<\text { tol. }
$$

The sequence $\left\{\delta \boldsymbol{u}^{k+1}, \delta p^{k+1}\right\}_{k}$ ought converge to $\left(\boldsymbol{u}^{n+1}-\boldsymbol{u}^{n}, p^{n+1}-p^{n}\right)$. Obviously, the cost of each nonlinear iteration is the cost of one residual evaluation and a number of solutions to the linearized subproblems.

\section{NUMERICAL EXAMPLES}

As a sample of our results, we present simulations corresponding to the general systems (2.11) and (2.14) in different scenarios. Our finite element solver is based on the open source C++ object oriented parallel library LifeV [32] (and 2D computations are carried out using a FreeFem++ [33] code). As stated in the previous section, we approximate the displacement field $\boldsymbol{u}$ with $\mathbb{P}_{2}$ finite elements, whereas for the pressure $p$ and the electrical potential fields $v, u_{\mathrm{i}}, u_{\mathrm{e}}, \boldsymbol{w}$, we use piecewise linear elements. Our main objective now reduces to provide a qualitative insight of the main features of the model. Even if the fibers' distribution is rather known (subepicardial myofibers follow a left-handed helix parallel to the wall, crossing the wall near the apex, and then continue in a righthanded helical pathway at the subendocardium; the fibers cross over to the subepicardium near the base), some simplifications can be assumed, for instance, that fibers are aligned with a fixed angle (as will be carried out for the 2D example in the succeeding sections).

\subsection{A single fiber simulation}

To investigate the propagation of a depolarization wave in a moving domain, we considered the system (2.14) for $t>0$ and $X \in \Omega_{o}=(0,1)$. In one spatial dimension, it reduces to solving the following parabolic PDE system: 


$$
\begin{aligned}
\partial_{t}\left(v\left(1+\gamma_{1}\right)\right)-D \partial_{X}\left(\left(1+\gamma_{1}\right)^{-1} \partial_{X} v\right) & =\left(1+\gamma_{1}\right) I_{\text {ion }}(v, w) \\
\partial_{t} w & =H(v, w),
\end{aligned}
$$

endowed with no-flux boundary conditions. Here, $I_{\text {ion }}$ and $H$, when taken as in (2.3), assume the following adimensional parameters [13] $a=0.13, b=0.013, c_{1}=0.26, c_{2}=0.1, D=5.6 \times 10^{-3}$. With this choice of parameters, the traveling waves produced have positive speed. Moreover, the contraction model here corresponds to (2.8). Impulse propagation was initiated by application of a stimulus current to the leftmost part of the fiber. The Luo-Rudy I kinetics along with the contraction model (2.9) are also used in this first test. In Figure 4, we display the time evolution of the transmembrane potential (species $v$ ) until the time $t=300 \mathrm{~ms}$, at the same reference point in a contractile and fixed fiber (left), and a snapshot of the spatial distribution of $v$ in $\Omega_{o}$ at a fixed time $t=80 \mathrm{~ms}$ (right). The dashed lines correspond to the propagation of the transmembrane potential on a fixed fiber, whereas the solid line represents its counterpart in a coupled propagation-contraction of the domain. The representation is carried out in the $X$-coordinates. The situation for both RogersMcCulloch and Luo-Rudy models is depicted (top and bottom of Figure 4, respectively). From the time evolution plots (left column), it is observed that the electrical potential in a fixed reference point has an earlier variation in a contractile fiber than in a fixed one. This is well in agreement with previous works (as, e.g., [34]). Moreover, the snapshots on the right column show that (a pull back to the reference configuration of) the potential wave in a contractile domain travels faster than that on the fixed domain. We stress that this behavior does not imply that in our model the mechanical response precedes the electrical propagation.
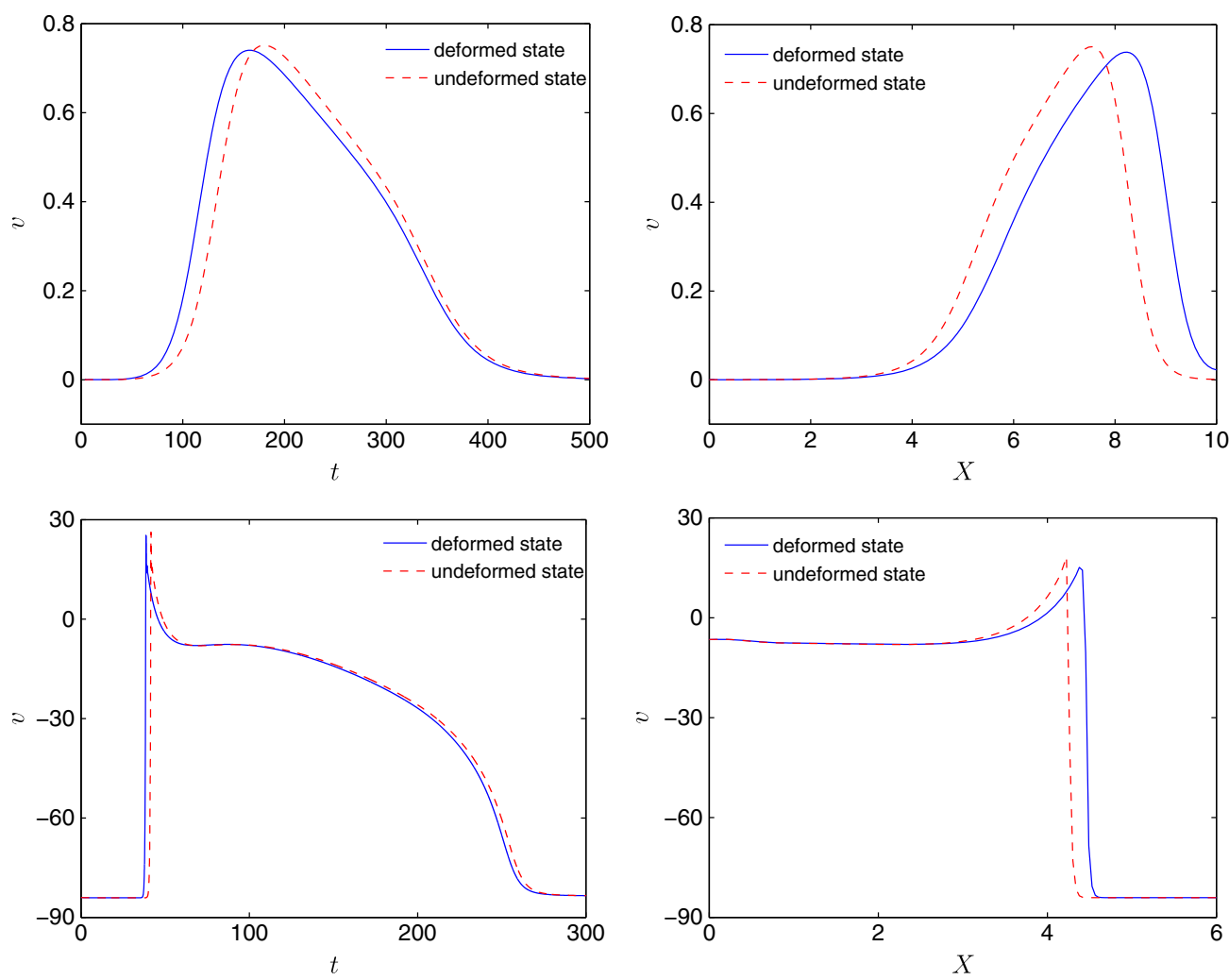

Figure 4. 1D Rogers-McCulloch (top row) and Luo-Rudy (bottom row) monodomain models: time evolution of the membrane potentials (left column) in the pure electrical propagation (dashed lines) and in the coupling with a description for the contraction of the fiber (solid lines), and potentials' distribution over the fiber (right column). 


\subsection{A $2 D$ slab of tissue}

In order to validate our mechanical numerical scheme (following [31]), we perform one time-step iteration, so that all potential fields are known constant quantities acting as initial conditions. The system to solve corresponds to (2.14) on the spatial domain $\Omega_{o}=(-1,1)^{2}$. A stretching in the $X_{1}$-direction is assumed, along with a compression in the $X_{2}$-direction. Let us define $\lambda=1+\beta X_{1}$. The given body force and boundary data (zero displacements on the bottom and traction on the remaining edges of the slab) are chosen such that the solution of the mechanical problem is $\boldsymbol{u}=\left(\beta X_{1}^{2} / 2, X_{2}\left(1+\beta X_{1}\right)^{-1}-X_{2}\right)^{T}, p=\mu_{1} / 2$, which gives

$$
\mathbf{F}=\left[\begin{array}{cc}
1+\beta X_{1} & 0 \\
-X_{2}\left(1+\beta X_{1}\right)^{-2} & \left(1+\beta X_{1}\right)^{-1}
\end{array}\right]
$$

and satisfies the incompressibility condition.

Analogously, we perform a validation of the electrical solver, taking only the monodomain Rogers-McCulloch problem (see, e.g., [5]). Non-homogeneous Dirichlet boundary conditions are imposed on the boundaries of the unit square, $v\left(X_{1}, X_{2}, t\right)=k(t)$, where $k(\cdot)$ and the model parameters are chosen such that the problem possesses the following analytical solution

$$
v\left(X_{1}, X_{2}, t\right)=\left\{1+0.001 \exp \left(\sqrt{1 / 2}\left(X_{1}-b_{0} t\right)\right)\right\}^{-1} .
$$

Figure 5 displays the error history for both model problems. We see that (left plot) a convergence of order $h^{2}$ is recovered for the displacements in the $H^{1}$-norm, whereas a cubic rate of convergence is achieved in the $L^{2}$-norm. For the pure electric problem, a linear convergence is obtained for the electrical potential.

We now consider the anisotropic monodomain electromechanical problem (2.14) on the unit square. We assume that the fibers are aligned with the $X_{1}$-axis, so that the conductivity tensor is a diagonal matrix of entries (in $\Omega^{-1} \mathrm{~cm}^{-1}$ ) $D_{11}=\sigma_{1}=3.28 \times 10^{-2}, D_{22}=\sigma_{\mathrm{t}}=6.99 \times 10^{-3}$. The membrane model used is the rescaled Rogers-McCulloch (2.4), for which the remaining parameters are $T=0.63 \mathrm{~ms}, A=130 \mathrm{mV}, v_{0}=v_{\min }=-84.0 \mathrm{mV}$. The initial data for the transmembrane potential and gating variable are

$$
v_{0}\left(X_{1}, X_{2}\right)=1-\left(1+\exp \left(-50 \sqrt{X_{1}^{2}+\left(X_{2}-0.5\right)^{2}}\right)\right), \quad w_{0}\left(X_{1}, X_{2}\right)=0 .
$$

The portion of the boundary where homogeneous Dirichlet boundary conditions for the displacement field are applied is the bottom edge, whereas the other edges experience no externally applied force. The elastic and bulk moduli are $\mu_{1}=4, \mu_{2}=8.5$, and the activation parameters are $\beta=0.3$, $v_{\max }=26$. The domain is discretized using 4406 vertices ( 8570 triangles), and the time evolution
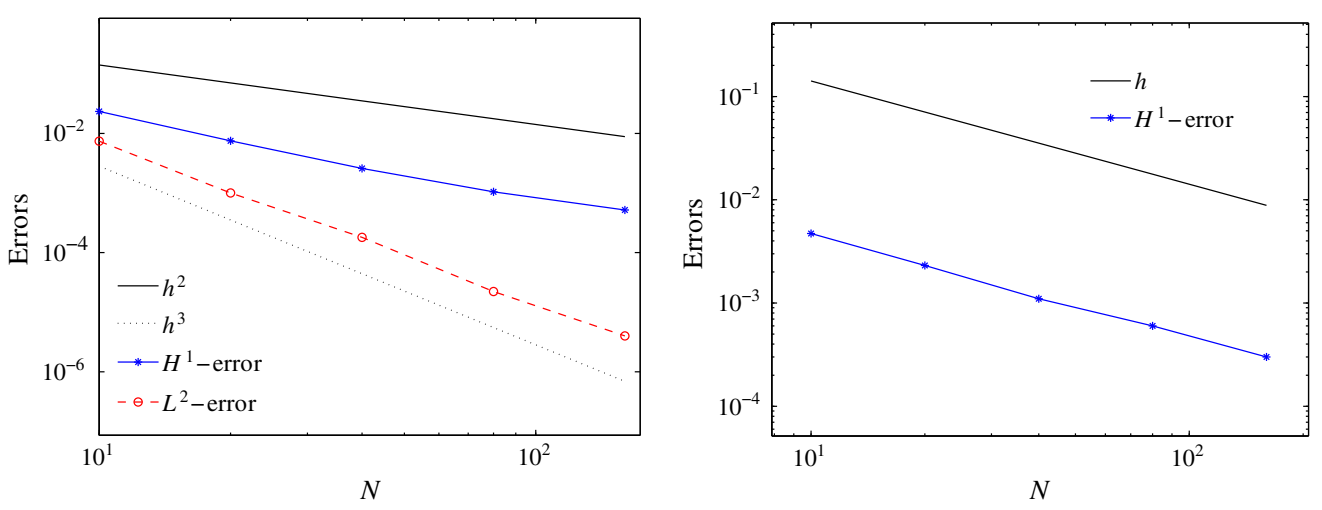

Figure 5. Convergence history (left) for the displacements in a pure mechanical model problem and (right) for the electrical potential in a pure electrical model problem, on a 2D slab. 
parameters are set to $T_{\text {final }}=600 \mathrm{~ms}, \Delta t=1 \mathrm{~ms}$. A tolerance of $1.0 \times 10^{-5}$ is used for the Newton stopping criterion (3.6), achieving convergence almost always in less than five iterations.

Several experiments have been conducted to obtain a qualitative comparison between the behaviors of the incompressible and nearly compressible formulations. Figure 6 displays snapshots at three different time instants of the potential field and corresponding deformed domain for both models, plus pressure isovalues for the incompressible formulation. As in the 1D simulations, it is observed that the propagation of the electrical wave induces contraction of the tissue. Although the results are qualitatively different (in terms of magnitude of the transmembrane potential and displacements), we see that the shown deformations generate strains of reasonable magnitude in both cases, whereas the pressure field shows no spurious oscillations.

Moreover, we noticed that with a compressible model, the displacements in the fibers direction are less pronounced than in the incompressible case. This can be readily seen from Figure 7, where
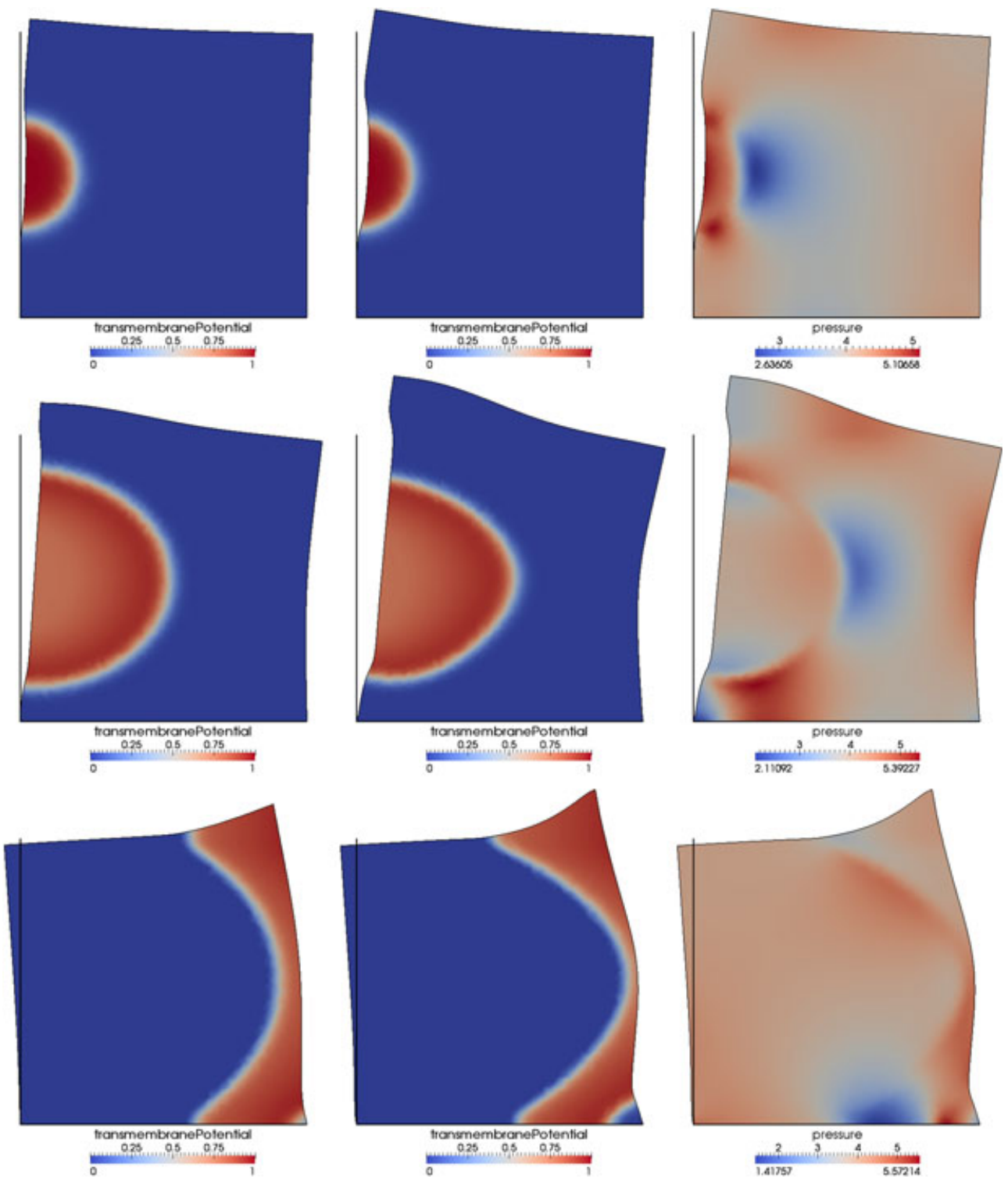

Figure 6. Rogers-McCulloch anisotropic monodomain electromechanical coupling: time evolution of the transmembrane potential and deformed configuration (compared with the resting state) for the compressible model (left), incompressible model (center), and pressure isovalues, for time instants $t=2 \mathrm{~ms}$ (top), $t=200 \mathrm{~ms}$ (middle), and $t=400 \mathrm{~ms}$ (bottom). 


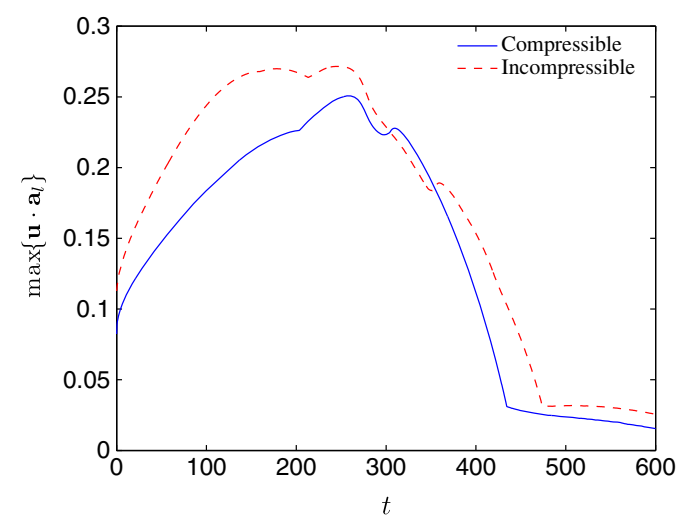

Figure 7. Rogers-McCulloch anisotropic monodomain electromechanical coupling: maximum displacement in the fibers direction $\max \left(\boldsymbol{u} \cdot \boldsymbol{a}_{1}\right)$ for a compressible (solid line) and an incompressible (dashed line) Neo-Hookean slab.
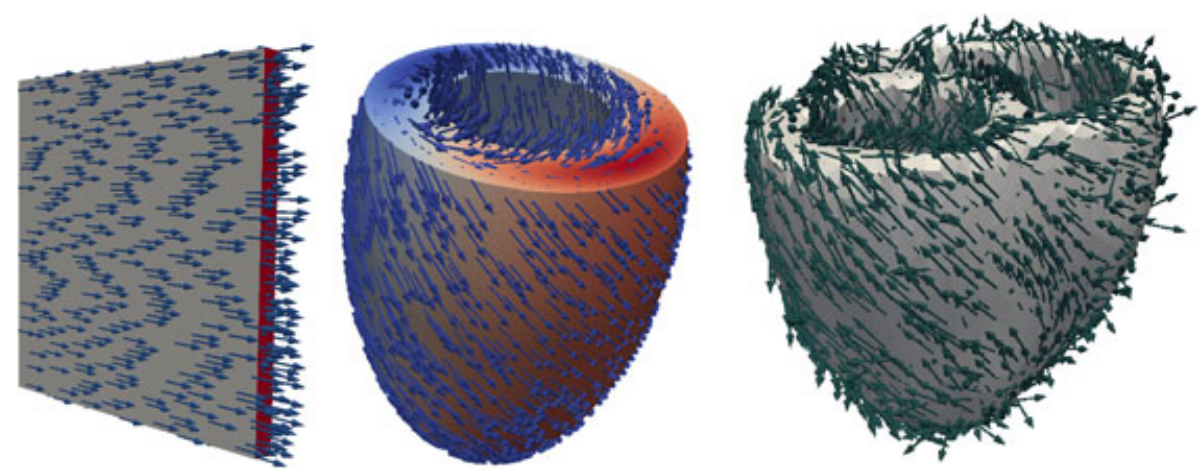

Figure 8. Orientation of muscle fibers for a slab, an idealized left ventricle, and a biventricular geometry.

we have plotted the maximum absolute displacements in the fibers direction versus time. The difference between these displacements can be tuned by modifying the bulk modulus $\mu_{2}$. Regarding ventricular twist and torsion, previous studies [35] suggest that a compressible model is able to better reproduce the experimental data.

\subsection{Test on a truncated ellipsoid}

Now we illustrate the behavior of the electromechanical coupled model on a idealized left ventricle of height $10 \mathrm{~cm}$, discretized using 29,560 nodes forming 155,770 tetrahedra. For our choice of finite elements, this implies that we handle 222,556 degrees of freedom for the displacements and 29,560 degrees of freedom for each electrical potential field and gating variable. The domain is initially subject to a periodic pure electrical external stimulus. The time discretization parameters are set to $T_{\text {final }}=600 \mathrm{~ms}, \Delta t=0.5 \mathrm{~ms}$, and the Luo-Rudy kinetics along with the bidomain model are used to describe the electrical activity. For the orientation of the cardiac fibers, we employ an analytical description given in, for example, [6] (Figure 8).

To initiate the excitation, we assume that the tissue is polarized at the beginning of cardiac cycle. This means that ionic variables are set to zero, whereas the transmembrane potential corresponds to constant resting state $v_{0}=-84.0 \mathrm{mV}$, and we impose an initial stimulus of magnitude $100 \mathrm{mV}$ on a thin region in the inner ellipsoid, representing a subendocardial layer of the ventricle. The region where the spread of excitation is initiated is given by $R=$ $\left\{\left(X_{1}, X_{2}, X_{3}\right) \in \Omega:\left(X_{1}-a_{1}\right)^{2} / R_{1}^{2}+\left(X_{2}-a_{2}\right)^{2} / R_{2}^{2}+\left(X_{3}-a_{3}\right)^{2} / R_{3}^{2} \leqslant 1\right\}$, where $a_{1}=a_{2}=$ $0, a_{3}=-0.15, R_{1}=R_{2}=1.75$, and $R_{3}=4.6$. The conductivity parameters (in $\Omega^{-1} \mathrm{~cm}^{-1}$ ) are $\sigma_{i}^{1}=3 \times 10^{-3}, \sigma_{i}^{\mathrm{t}}=3.1525 \times 10^{-4}, \sigma_{e}^{1}=2 \times 10^{-3}, \sigma_{e}^{\mathrm{t}}=1.3514 \times 10^{-3}$. The elastic and 
activation parameters are set as in the previous subsection. Essential boundary conditions on the top plane (representing the base of the ventricle) are imposed $(\boldsymbol{u} \cdot \boldsymbol{n}=0)$, whereas on the rest of $\partial \Omega$, we specify $\mathbf{P} \boldsymbol{n}=\mathbf{0}$.

For this example, we employ the nearly incompressible electromechanical model. From Figure 9, and analogously to the $1 \mathrm{D}$ and $2 \mathrm{D}$ simulations, a propagation of the electrical wave is observed, which induces contraction of the cardiac tissue.

Figure 10 examines the scaling of the solver for the Luo-Rudy bidomain electromechanical model on a refined ellipsoid (39,850 vertices) at time instant $t=60 \mathrm{~ms}$ (when the physics of the coupled problem is already clearly noticeable). The figure provides results in terms of the number of linear iterations, the average CPU timing for each linear iteration, each preconditioner computation (built using two layers of overlap), and a single time step. Up to 128 processors, the algorithm shows a reasonable scalable behavior. As the preconditioner computation plays a major part of the overall process, we re-use it. We further stress that, for our specific choice of space discretization and number of degrees of freedom (even for several meshes with different mesh sizes), the mechanical solver takes roughly $70 \%$ of the total runtime for the solution algorithm. This difference in required computational effort obeys to the use of the same mesh for the discretization of the mechanical and electrical systems. The mentioned percentage depends also on the underlying membrane model (when using Luo-Rudy I, a slightly lower percentage of the overall CPU time is spent on the Newton method for the mechanical problem), but in general, the ODE systems for the membrane and activation function are almost perfectly scalable, as there is no diffusion involved.

\subsection{Test on a biventricular geometry}

Finally, we perform several numerical tests by using a 3D biventricular geometry, which contains a description of the fibers' distribution obtained via a diffusion tensor statistical analysis [36]. The associated tetrahedral mesh consists of 6598 vertices and 30,309 elements. The simulation corresponds to the phase preceding atrial contraction; therefore, the reference state coincides with a stress-free configuration. The bidomain Rogers-McCulloch electromechanical model with
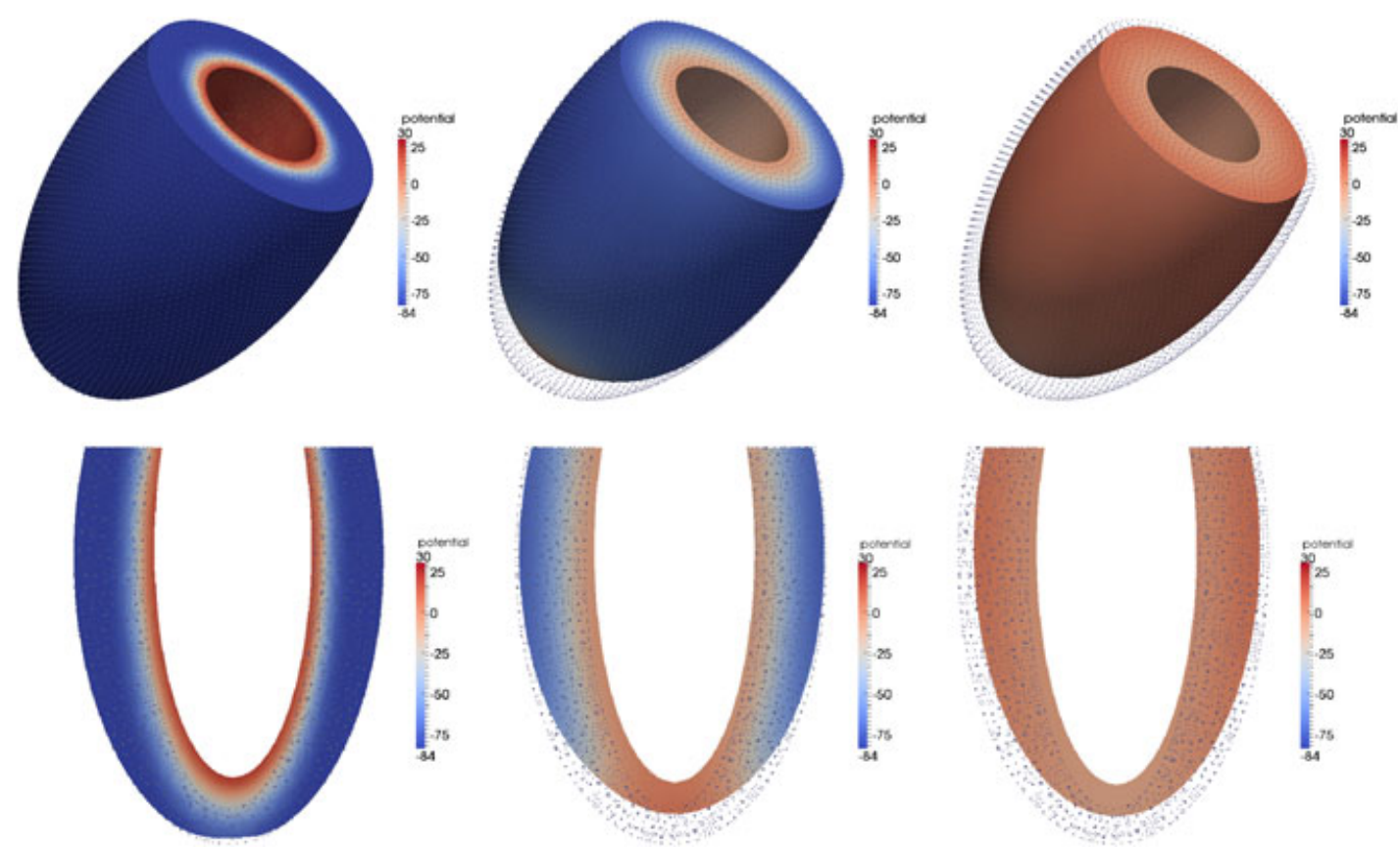

Figure 9. Snapshots of the evolution of the transmembrane potential and corresponding movement of the mesh, Luo-Rudy I, bidomain electro-mechanic model on a truncated ellipsoid at time instants $t=$ 6,200,600 ms (left, middle, right, respectively). The undeformed geometry is represented by a cloud of points. 

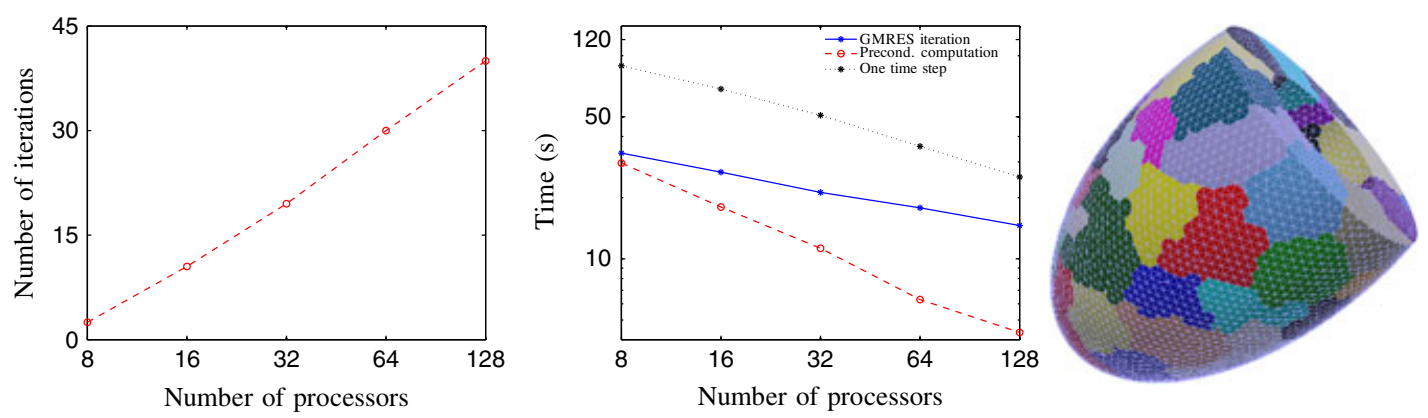

Figure 10. Scalability results for the Luo-Rudy bidomain electro-mechanic model at time instant $t=60 \mathrm{~ms}$. Number of linear iterations (left), time spent on different parts of the algorithm (middle), and mesh partition into 64 sub-domains of a truncated ellipsoid (right).
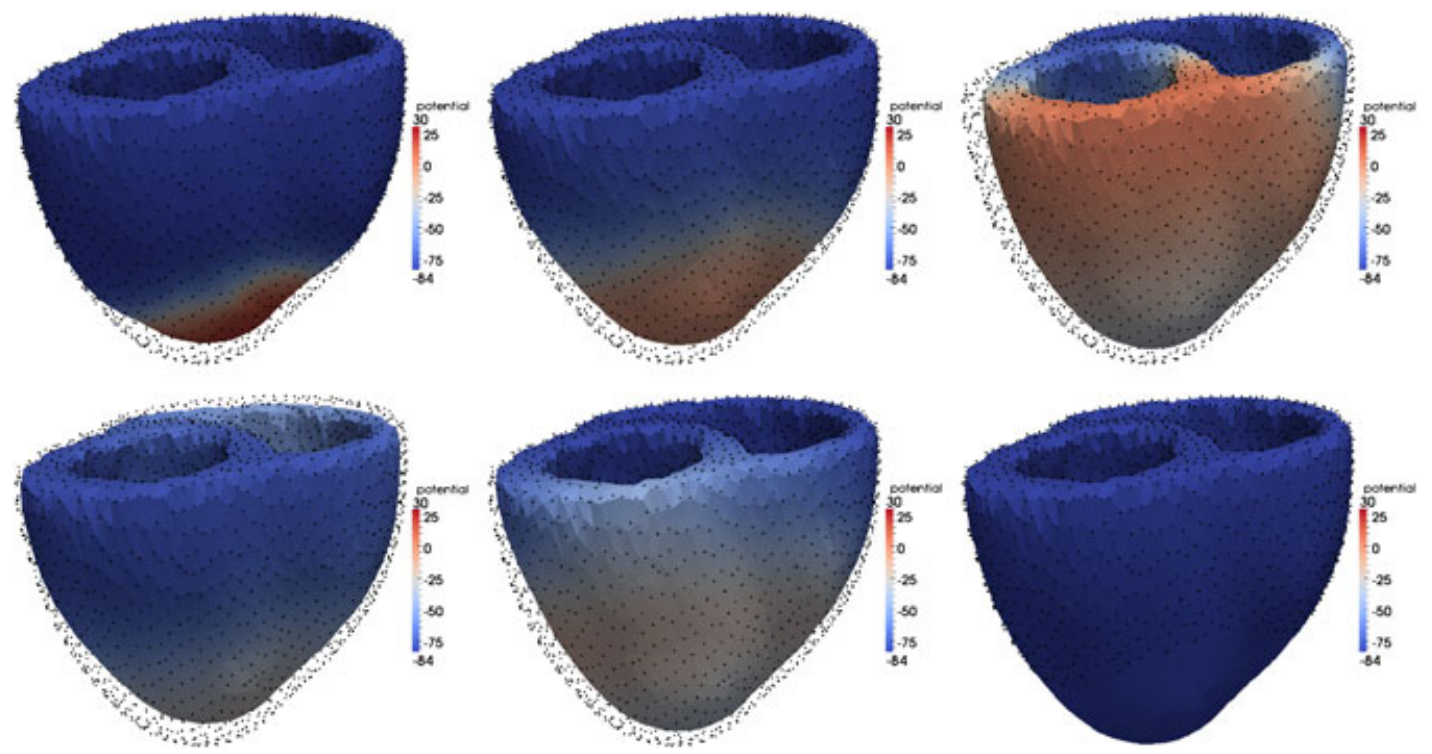

Figure 11. Snapshots of the evolution of the transmembrane potential and corresponding movement of the mesh, Rogers-McCulloch monodomain electro-mechanic model at time instants $t=10,40$, $100,300,500,600 \mathrm{~ms}$ (from top-left).

activation governed by (2.10) is studied here, and Figure 11 illustrates the propagation of the transmembrane potential front, along with the displacement field through the cardiac muscle. After the initial activation, a second activation stimulus is applied at $t=400 \mathrm{~ms}$. Already at $t=10 \mathrm{~ms}$, one observes a displacement of the apex induced by the initial impulse, followed by an overall contraction of the myocardium with an increased intensity in the zones where the wave of electrical excitation assumes high values. As for boundary data, reflecting conditions have been imposed for the electrical potentials everywhere, whereas for displacements, as it is known that a portion of the right ventricle connecting with the pulmonary artery remains practically fixed during contraction [37], we have specified no displacement on a region of the right ventricular endocardial base. Qualitatively similar results are obtained (Figure 12) after imposing Robin boundary conditions $\mu_{1} \boldsymbol{u}+\mathbf{P} \boldsymbol{n}=\mathbf{0}$ on the epicardial and basal boundaries. As discussed in, for example, [38] for the simulation of arteries, this setting may be regarded as an elastic resistance of the tissues surrounding the myocardium, being proportional to the displacement. This permits a slight contraction of the base, in contrast with no displacement in the previous alternative.

Of course, taking into account the particular structure of the extramyocardial region would provide a more realistic setting, as it is known that the heart moves within the pericardium sack and 

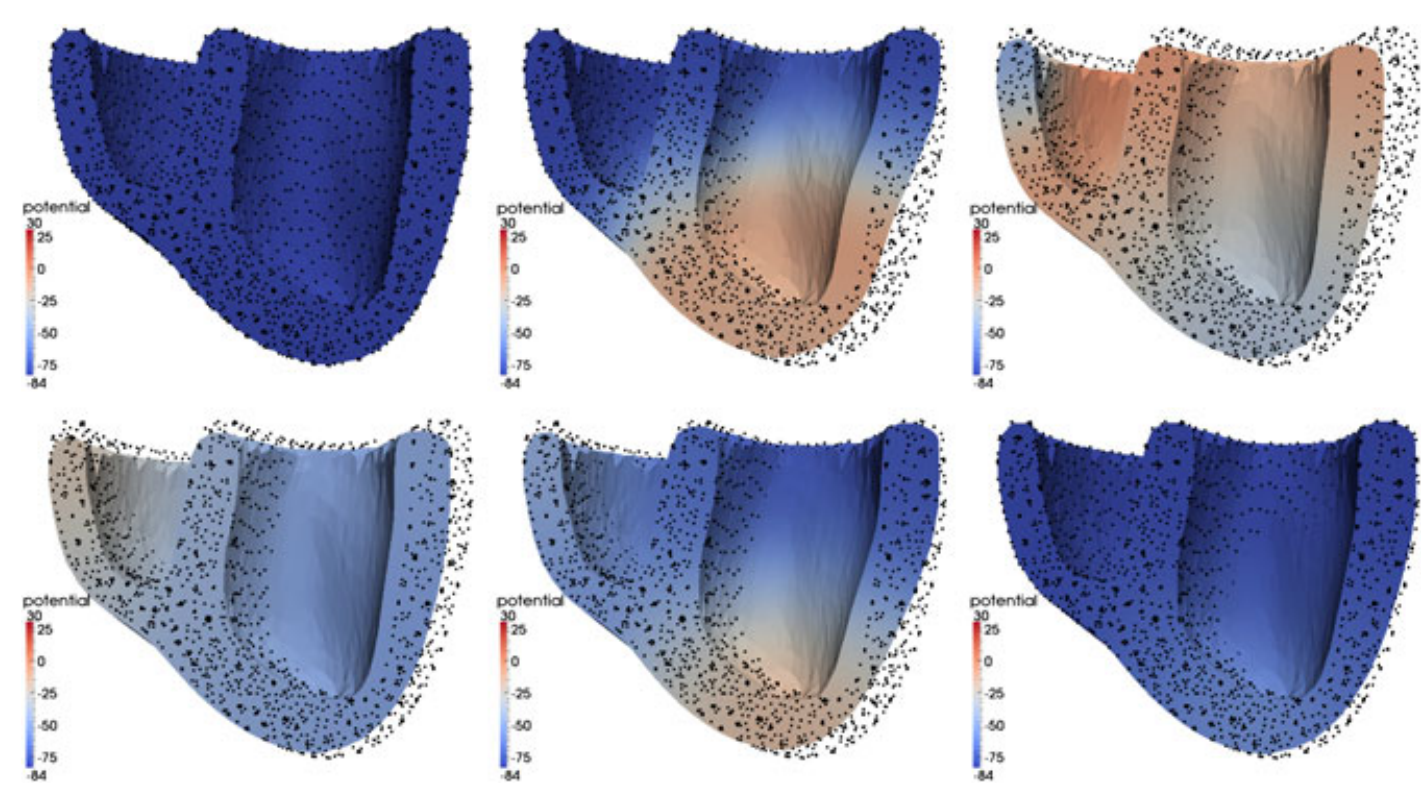

Figure 12. Snapshots of the evolution of the transmembrane potential and corresponding movement of the mesh, Rogers-McCulloch monodomain electro-mechanic model at time instants $t=0$, 90, 180, 300, 500, $600 \mathrm{~ms}$ (from top-left).

is constrained also by the other connecting vessels. Detailed expressions for physiologically relevant boundary conditions are provided in [22], and a deep discussion on some mathematical and numerical implications of choosing appropriate boundary conditions is presented in [39].

\section{CONCLUSION}

In this paper, we have proposed a numerical method for a coupled electromechanical model of the cardiac tissue. The electrical part of the model includes a description of the anisotropy in the medium and allows for different electric membrane models. The interaction between the electric and mechanical activity is taken into account by assuming that when decomposing multiplicatively the visible deformation, its active part depends directly on the transmembrane potential through a saturation-like function. In this active part, we also include the anisotropic description of the tissue. The FEM is based on $\mathbb{P}^{2}$ elements for the displacements, whereas for electrical potentials and pressure field, we use $\mathbb{P}^{1}$ elements.

From the modeling point of view, we stress that a deeper understanding of the electromechanical coupling is still needed and open to discussion. As present limitations in our treatment, we have neglected several aspects in the modeling of the heart function, such as an accurate anatomical representation of the fiber directions, a model for the blood circulation, Starling effects, a more involved model for the passive mechanical properties of the medium, and ionic-scale electromechanics (intracellular calcium handling and cross-bridge formation). Also, the quantitative validation of the model used here implies a difficult task due to the lack of sufficient experimental data. Nevertheless, this work is being extended to consider some of these aspects for current and future studies, with special focus on cases of real pathological interest. To mention an important example, cardiac heterogeneities could cause electrical impulses originating from one area of tissue to fail to conduct into areas with prolonged repolarization [21,40]. It is also known that under dilated myocardiopathy, which is a common pathology, the electrical potential is able to propagate through all the tissue, whereas there are specific regions of the ventricular chamber where no contraction takes place. This is a clear application in which cardiac electromechanical models have a special interest over pure electrophysiological models. 
From the numerical viewpoint, several improvements can be rather straightforwardly included in the proposed method. First of all, the time-stepping strategy could be upgraded to an adaptive scheme by using a similar algorithm as the one proposed in [6], where the use of a small time step in the excitation phase would increase the accuracy in capturing the action potential upstroke, whereas a large time step could be used for the plateau phase. Secondly, other finite element discretizations and more sophisticated preconditioning algorithms are sought, such as the monodomain-based block-triangular preconditioning proposed in [7] or structured algebraic multigrid preconditioners in the spirit of [8].

Finally, to the authors' knowledge, the well-posedness analysis, global existence, regularity of solutions, and related questions concerning the mathematical study of cardiac electromechanical models have not been thoroughly addressed. Although a rigorous analysis in this direction is currently under development [28], we can anticipate that the main difficulty lies in treating the geometrical nonlinearity introduced in the electrical diffusion operator by the change of coordinates from Eulerian to Lagrangian. A possible way to circumvent this issue consists in considering a linearized contribution of the mechanical response on the bidomain equations, or alternatively, applying a truncation operator that eventually allows us to bound the coupling term $(\mathbf{I}+\nabla \boldsymbol{u})^{-1} \mathbf{D}_{k}(\mathbf{I}+\nabla \boldsymbol{u})^{-T}$.

\section{ACKNOWLEDGEMENTS}

The authors gratefully acknowledge the discussions with Davide Ambrosi regarding the active strain formulation. This work has been supported by the European Research Council through the advanced grant "Mathcard, Mathematical Modelling and Simulation of the Cardiovascular System", project ERC-2008-AdG 227058 .

\section{REFERENCES}

1. Cherubini C, Filippi S, Nardinocchi P, Teresi L. An electromechanical model of cardiac tissue: constitutive issues and electrophysiological effects. Progresses in Biophysics and Molecular Biology 2008; 97:562-573.

2. Nash MP, Panfilov AV. Electromechanical model of excitable tissue to study reentrant cardiac arrhythmias. Progresses in Biophysics and Molecular Biology 2004; 85:501-522.

3. Pathmanathan P, Whiteley JP. A numerical method for cardiac mechanoelectric simulations. Annals of Biomedical Engineering 2009; 37:860-873.

4. Sainte-Marie J, Chapelle D, Cimrman R, Sorine M. Modeling and estimation of the cardiac electromechanical activity. Computers and Structures 2006; 84:1743-1759.

5. Bendahmane M, Bürger R, Ruiz-Baier R. A multiresolution space-time adaptive scheme for the bidomain model in electrocardiology. Numerical Methods for Partial Differential Equations 2010; 26:1377-1404.

6. Colli Franzone P, Pavarino LF. A parallel solver for reaction-diffusion systems in computational electro-cardiology. Mathematical Models and Methods in Applied Sciences 2004; 14:883-911.

7. Gerardo-Giorda L, Mirabella L, Nobile F, Perego M, Veneziani A. A model-based block-triangular preconditioner for the bidomain system in electrocardiology. Journal of Computational Physics 2009; 228:3625-3639.

8. Pennacchio M, Simoncini V. Algebraic multigrid preconditioners for the bidomain reaction-diffusion system. Applied Numerical Mathematics 2009; 59:3033-3050.

9. Ambrosi D, Arioli G, Nobile F, Quarteroni A. Electromechanical coupling in cardiac dynamics: the active strain approach. SIAM Journal of Applied Mathematics 2011; 71:605-621.

10. Nardinocchi P, Teresi L. On the active response of soft living tissues. Journal of Elasticity 2007; 88:27-39.

11. Ravens U. Mechano-electric feedback and arrhythmias. Progresses in Biophysics and Molecular Biology 2003; 82:255-266.

12. Tung L. A bi-domain model for describing ischemic myocardial D-C currents. PhD thesis, MIT, Cambridge, MA, 1978.

13. Rogers JM, McCulloch AD. A collocation-Galerkin finite element model of cardiac action potential propagation. IEEE Transactions on Biomedical Engineering 1994; 41:743-757.

14. Luo C, Rudy Y. A model of the ventricular cardiac action potential: depolarization, repolarization, and their interaction. Circulation Research 1991; 68:1501-1526.

15. Bürger R, Ruiz-Baier R. Adaptive multiresolution simulation of waves in electrocardiology. In Numerical Mathematics and Advanced Applications. Kreiss G, et al (eds). Springer-Verlag: Berlin Heidelberg, 2010; 199-207.

16. Holzapfel GA, Ogden RW. Constitutive modelling of passive myocardium: a structurally based framework for material characterization. Philosophical Transactions of the Royal Society A 2009; 367:3445-3475.

17. Simo JC, Pister KS. Remarks on rate constitutive equations for finite deformations. Computational Methods in Applied Mechanics and Engineering 1984; 46:201-215.

18. Taber LA, Perucchio R. Modeling heart development. Journal of Elasticity 2000; 61:165-197. 
19. Nash MP, Hunter PJ. Computational mechanics of the heart. Journal of Elasticity 2000; 61:113-141.

20. Ciarlet PG. Mathematical Elasticity, Vol. 1. Three Dimensional Elasticity. North-Holland: Amsterdam, 1998.

21. Kerckhoffs R, Bovendeerd P, Kotte JC, Prinzen F, Smiths K, Arts T. Homogeneity of cardiac contraction despite physiological asynchrony of depolarization: a model study. Annals of Biomedical Engineering 2003; 31:536-547.

22. Usyk TP, LeGrice IJ, McCulloch AD. Computational model of three-dimensional cardiac electromechanics. Computing and Visualization in Science 2002; 4:249-257.

23. Rossi S, Ruiz-Baier R, Pavarino LF, Quarteroni A. Active strain and activation models in cardiac mechanics. Submitted.

24. Rice JJ, Wang F, Bers DM, de Tombe PP. Approximate model of cooperative activation and crossbridge cycling in cardiac muscle using ordinary differential equations. Biophysical Journal 2008; 95:2368-2390.

25. Colli Franzone P, Savaré G. Degenerate evolution systems modeling the cardiac electric field at micro- and macroscopic level. In Evolution Equations, Semigroups and Functional Analysis, Lorenzi A, Ruf B (eds). Birkhäuser: Basel, 2002; 49-78.

26. Veneroni M. Reaction-diffusion systems for the macroscopic Bidomain model of the cardiac electric field. Nonlinear Analysis: Real World Applications 2009; 10:849-868.

27. Krejčí P, Sainte-Marie J, Sorine M, Urquiza JM. Solutions to muscle fiber equations and their long time behaviour. Nonlinear Analysis: Real World Applications 2006; 7:535-558.

28. Andreianov B, Bendahmane M, Quarteroni A, Ruiz-Baier R. Mathematical analysis of a coupled model in cardiac electromechanics. Submitted.

29. Göktepe S, Kuhl E. Electromechanics of the heart: a unified approach to the strongly coupled excitation-contraction problem. Computational Mechanics 2010; 45:227-243.

30. Quarteroni A. Numerical models for differential problems, MS\&A series, Vol. 2. Springer-Verlag: Milan, 2009.

31. Pathmanathan P, Gavaghan D, Whiteley JP. A comparison of numerical methods used for finite element modelling of soft tissue deformation. Journal of Strain Analysis 2009; 44:391-406.

32. LifeV library. http://www.lifev.org.

33. Hecht F. FREEFEM++, 3rd edn. Université Pierre et Marie Curie, Laboratoire Jacques-Louis Lions: Paris, 2008.

34. Vetter FJ, Rogers JM, McCulloch AD. A finite element model of passive mechanics and electrical propagation in the rabbit ventricles. Computational Cardiology 1998; 25:705-708.

35. Taber LA, Yang M, Podzus WW. Mechanics of ventricular torsion. Journal of Biomechanics 1996; 29:745-752.

36. Peyrat JM, Sermesant M, Pennec X, Delingette H, Xu C, McVeigh ER, Ayache N. A computational framework for the statistical analysis of cardiac diffusion tensors: application to a small database of canine hearts. IEEE Transactions on Medical Imaging 2007; 26:1500-1514.

37. Gurev V, Lee T, Constantino J, Arevalo H, Trayanova NA. Models of cardiac electromechanics based on individual hearts imaging data. Biomechanics and modeling in mechanobiology 2011; 10:295-306.

38. Moireau P, Xiao N, Astorino M, Figueroa CA, Chapelle D, Taylor CA, Gerbeau JF. External tissue support and fluid-structure simulation in blood flows. Biomechanics and Modeling in Mechanobiology 2011, to appear.

39. Pathmanathan P, Chapman SJ, Gavaghan D, Whiteley JP. Cardiac electromechanics: the effect of contraction model on the mathematical problem and accuracy of the numerical scheme. Quarterly Journal of Mechanics and Applied Mathematics 2010; 63:375-399.

40. Trayanova NA. Whole-heart modeling: applications to cardiac electrophysiology and electromechanics. Circulation Research 2011; 108:113-128. 